\title{
Laplace-Domain, High-Order Homogenization for Transient Dynamic Response of Viscoelastic Composites
}

\author{
Tong Hui and Caglar Oskay* \\ Department of Civil and Environmental Engineering \\ Vanderbilt University \\ Nashville, TN 37235
}

\begin{abstract}
This manuscript presents a high order homogenization model for wave propagation in viscoelastic composite structures. Asymptotic expansions with multiple spatial scales are employed to formulate the homogenization model. The proposed multiscale model operates in the Laplace domain allowing the representation of linear viscoelastic constitutive relationship using a proportionality law. The high order terms in the asymptotic expansion of response fields are included to reproduce micro-heterogeneity induced wave dispersion and formation of bandgaps. The first and second order influence functions and the macroscopic deformation are evaluated using the finite element method with complex coefficients in the Laplace domain. The performance of the proposed model is assessed by investigating wave propagation characteristics in layered and particulate composites, and verified against direct numerical simulations and analytical solutions. The analysis of dissipated energy revealed that material dispersion may contribute significantly to wave attenuation in dissipative composite materials. The wave dispersion characteristics are shown to be sensitive to microstructure morphology.

Keywords: homogenization, bandgap, wave dispersion, Laplace transform, viscoelastic behavior.
\end{abstract}

\section{Introduction}

Wave propagation in heterogeneous media introduces complicated dynamic phenomena due to the reflections and refractions that occur at constituent material interfaces. Wave dispersion in heterogeneous materials has been routinely observed in many engineering applications that involve various waves including electro-magnetic, optical and acoustic waves. Material dispersion (i.e., dispersion introduced by material heterogeneity) becomes prominent when wavelength approaches the microstructural length scale, which allows interactions to take place. In addition, different waves usually coexist within the same structural domain and interact with each

*Corresponding author address: VU Station B\#351831, 2301 Vanderbilt Place, Nashville, TN 37235. Email: caglar.oskay@vanderbilt.edu 
other. The wave dispersion due to micro-heterogeneities and the wave interactions influence the propagation characteristics in terms of wave velocity, wave shape and wavenumber.

Dispersion and dispersion-induced dynamic response phenomena have been subject to a number of investigations particularly on composites with elastic constituents. The realization and modeling of these phenomenon dates back to the classical works of Cosserat and Cosserat [5], Mindlin [17], Eringen and Suhubi [9]. Material dispersion in composites has been modeled using a number of approaches including gradient elasticity [7], mathematical homogenization [13], Floquet-Bloch expansions [26], scale bridging through Hamilton's principle [34], and Mindlin's theory $[8,10]$. The dispersion relation between wave frequency and wavenumber in the frequency domain displays a banded structure with alternating passbands and stopbands. Stopbands refer to frequency bands within which the wave propagation is completely blocked. Band structures were found in the electromagnetic range (i.e., photonic bandgaps) [36, 35] as well as in the acoustic range (i.e., phononic bandgaps) $[27,14,33]$. In order to reveal the evanescent wave fields and bandgap structure, both the real and imaginary components of the wavenumber must be included [28]. Suzuki and Yu [29] and Andrianov et al. [2] employed the Floquet-Bloch theorem to model the appearance of bandgaps in photonics and phononic ranges, respectively. Hui and Oskay [13] proposed a multiscale homogenization model based on asymptotic expansion for wave propagation in elastic composite structures, which predicted bandgap formation and behavior up to and within the first stopband.

In composite materials with viscoelastic constituents, wave attenuation could be due to viscous dissipation, dispersive behavior or both. Studies on wave dispersion in viscoelastic composites are relatively scarce compared to elastic problems (see Refs. [23, 31, 25] for some recent investigations). Modeling research has typically focused on layered or lattice structures $[30,19,1,32,20]$ based on analytical and semi-analytical approaches. The literature on bandgap in viscoelastic composites is even more limited. Zhao and Wei [37] studied the bandgap of one-dimensional phononic crystal with viscoelastic host material using the BlochFloquet wave solution. Moiseyenko and Laude [18] investigated the influence of material loss on the complex band structure of two-dimensional phononic crystals by incorporating viscoelastic constitutive model in the extended plane-wave expansion. Psarobas [24] discussed the effect of viscoelastic losses in a high-density contrast sonic bandgap material of closed packed rubber sphere in air. Merheb et al. [16] provided a theoretical and experimental study of rubber/air acoustic bandgap structures. Oh et al. [21] investigated wave attenuation and dissipation mechanisms in viscoelastic phononic crystals having different inclusion types in a long-wavelength regime. Hui and Oskay [12] demonstrated the formation of bandgaps in a one-dimensional layered viscoelastic composite using a semi-analytical solution based on the multiscale homogenization theory. This work helps to elucidate the possibility of the high order homogenization model applied in viscoelastic materials. Available computational models capable of describing multi-dimensional wave propagation in viscoelastic composites including dispersion and bandgap phenomena appear very limited.

In this manuscript, a multi-dimensional, high order homogenization model for wave prop- 
agation in viscoelastic composite structures is presented. This model is formulated based on the method of asymptotic expansions with multiple spatial scales. The proposed formulation builds on the modeling approach presented in Ref. [13]. Three key novel contributions of the present manuscript are: (1) the proposed approach leads to a numerical model that can capture wave dispersion and behavior within the bandgaps of composites with viscoelastic constituents; (2) composite microstructures with arbitrary morphological complexity is possible since the proposed approach relies on the computational homogenization method; and (3) the asymptotic analysis is performed in the Laplace domain, which allows the representation of the linear viscoelastic constitutive relationship using a proportionality law. The performance of the proposed model is assessed by investigating wave propagation characteristics in layered and particulate composites, and verified against direct numerical simulations and analytical solutions. A method to compute the dissipated energy, including the microstructure effects, is provided. The analysis of dissipated energy revealed that material dispersion may contribute significantly to wave attenuation in dissipative composite materials at or near bandgap frequencies. The wave dispersion characteristics are shown to be sensitive to microstructure morphology.

The remainder of this manuscript is organized as follows: Section 2 describes the problem setting defined in the time domain and in the Laplace domain. Section 3 provides the formulation based on the mathematical homogenization theory with multiple spatial scales. Section 4 presents the calculation of dissipated energy using the high order homogenization model. Section 5 describes the strategy for the numerical implementation of the multiscale model. The numerical examples are discussed in Section 6. Section 7 provides the conclusions and the future research directions.

\section{Problem setting}

The illustrative description of multiscale problem is provided in Fig. 1. Let $\Omega$ denote the open bounded domain of the composite structure. The momentum balance under dynamic loading is expressed as:

$$
\sigma_{i j, j}^{\zeta}(\mathbf{x}, t)=\rho^{\zeta}(\mathbf{x}) \ddot{u}_{i}^{\zeta}(\mathbf{x}, t)
$$

where, $\boldsymbol{\sigma}^{\zeta}$ denotes the stress tensor; $\rho^{\zeta}$ the density; and $\mathbf{u}^{\zeta}$ the displacement vector. The superscript, $\zeta$, indicates that the response fields oscillate spatially due to the microstructural heterogeneity; $\mathbf{x}$ and $t$ denote the spatial coordinate vector and time coordinate, respectively. Superimposed dot indicates derivative with respect to time; and subscript comma denotes spatial derivative. The indicial notation follows the Einstein summation convention unless otherwise specified.

The constituents of the composite domain are taken to be linear viscoelastic, expressed using the hereditary integral:

$$
\sigma_{i j}^{\zeta}(\mathbf{x}, t)=\int_{0}^{t} g_{i j k l}^{\zeta}(\mathbf{x}, t-\tau) \dot{\epsilon}_{k l}^{\zeta}(\mathbf{x}, \tau) d \tau
$$




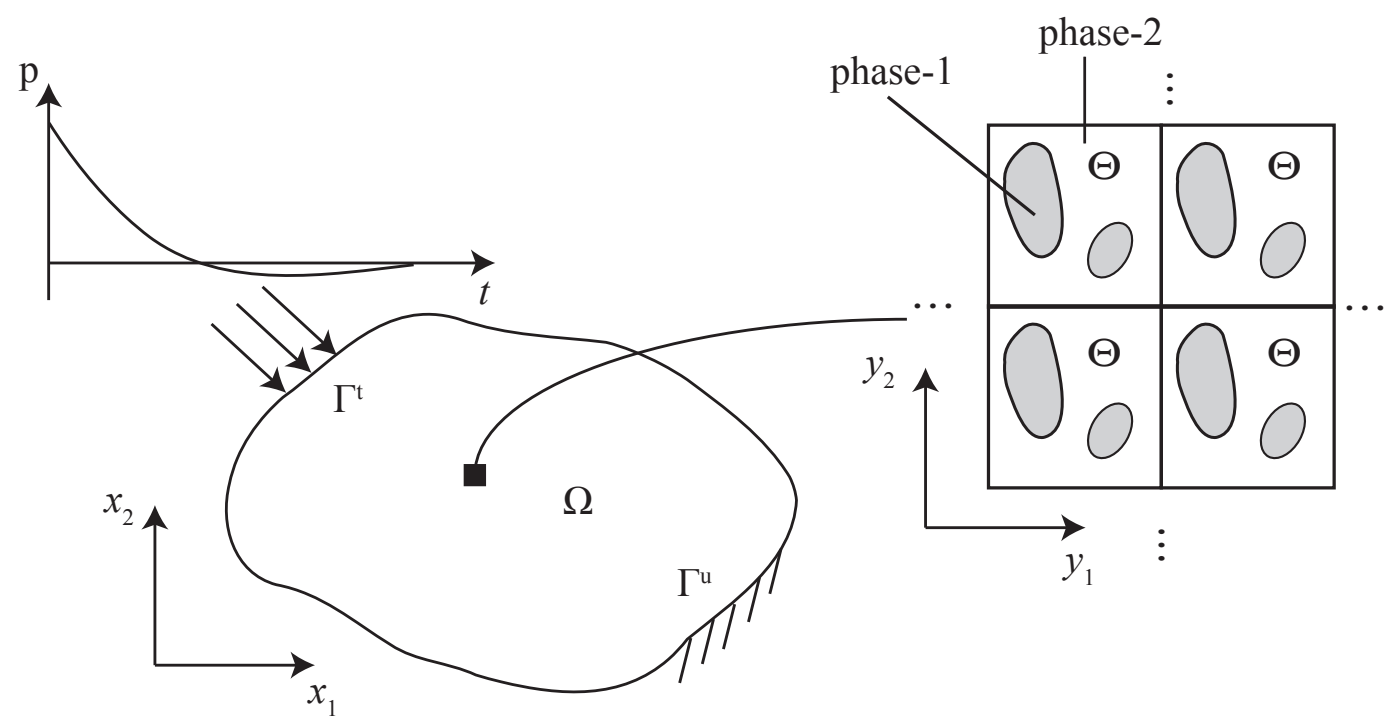

Figure 1: Schematic representation of multiscale problems.

in which, $\boldsymbol{\epsilon}^{\zeta}$ is the strain tensor and $\mathrm{g}^{\zeta}$ the time-varying relaxation moduli. Equation 2 indicates that the current stress state at a material point is a function of the entire strain history, but the constitutive response remains spatially local. For linear elastic materials, the standard linear-elastic constitutive law is recovered by taking $\mathbf{g}^{\zeta}$ independent of time and equal to the generalized Hooke's tensor. Under the assumption of small deformation:

$$
\epsilon_{i j}^{\zeta}(\mathbf{x}, t)=\frac{1}{2}\left(u_{i, j}^{\zeta}(\mathbf{x}, t)+u_{j, i}^{\zeta}(\mathbf{x}, t)\right)
$$

The boundary conditions are:

$$
\begin{gathered}
u_{i}^{\zeta}(\mathbf{x}, t)=\bar{u}_{i}(\mathbf{x}, t) ; \quad \mathbf{x} \in \Gamma^{u} \\
\sigma_{i j}^{\zeta}(\mathbf{x}, t) n_{j}=\bar{t}_{i}(\mathbf{x}, t) ; \quad \mathbf{x} \in \Gamma^{t}
\end{gathered}
$$

where, $\mathbf{n}$ denotes the outward unit normal vector along the traction boundaries; $\overline{\mathbf{u}}(\mathbf{x}, t)$ and $\overline{\mathbf{t}}(\mathbf{x}, t)$ are the displacement and traction data prescribed on $\Gamma^{u}$ and $\Gamma^{t}$, respectively. The boundary conditions are defined such that $\Gamma \equiv \partial \Omega=\Gamma^{u} \cup \Gamma^{t} ; \Gamma^{u} \cap \Gamma^{t}=\emptyset$. The initial displacement and velocity conditions are:

$$
\begin{aligned}
u_{i}^{\zeta}(\mathbf{x}, 0)=\hat{u}_{i}(\mathbf{x}) ; & \mathbf{x} \in \Omega \\
\dot{u}_{i}{ }^{\zeta}(\mathbf{x}, 0)=\hat{v}_{i}(\mathbf{x}) ; & \mathbf{x} \in \Omega
\end{aligned}
$$

in which, $\hat{\mathbf{u}}$ and $\hat{\mathbf{v}}$ denote the initial displacement and velocity data, respectively. In this work, homogeneous initial conditions are assumed for simplicity (i.e., $\hat{\mathbf{u}}=\hat{\mathbf{v}}=\mathbf{0}$ ). 


\subsection{Governing equations in the Laplace domain}

It is possible to proceed with the asymptotic expansions of the governing system of equations defined in the time domain (Eqs. 1-5) to formulate a multiscale homogenization model. From the computational perspective, this approach requires the evaluation and storage of separate microstructure problems at each integration point of a macroscale mesh. The form of the hereditary integral along with the momentum balance equation allows a simpler description of the problem when posed in the Laplace domain. In what follows, the governing initial boundary value problem is recast in the Laplace domain, and the key characteristics of the Laplace transform is provided.

The Laplace transform of an arbitrary, real valued, time varying function, $f$, is defined as:

$$
\mathscr{F}(s) \equiv \mathscr{L}(f(t))=\int_{0}^{\infty} e^{-s t} f(t) d t
$$

where, the Laplace argument, $s$, and the transformed function, $\mathscr{F}$, are complex valued. The derivative rule for the Laplace transform is given as:

$$
\mathscr{L}(\underbrace{t t \ldots t}_{n \text { times }}(t))=s^{n} \mathscr{F}(s)-s^{n-1} f(0)-\ldots-f \underbrace{t t \ldots t}_{n-1 \text { times }}(0)
$$

and the convolution integral rule is given as:

$$
\mathscr{L}\left(\int_{0}^{t} f_{1}(t-\xi) f_{2}(\xi) d \xi\right)=\mathscr{L}\left(\int_{0}^{t} f_{1}(\xi) f_{2}(t-\xi) d \xi\right)=\mathscr{L}\left(f_{1}\right) \mathscr{L}\left(f_{2}\right)
$$

Applying Eq. 8 to Eq. 2, the constitutive equation in the time domain is transformed to the proportional form in the Laplace domain:

$$
\sigma_{i j}^{\zeta}(\mathbf{x}, s)=C_{i j k l}^{\zeta}(\mathbf{x}, s) \epsilon_{k l}^{\zeta}(\mathbf{x}, s)
$$

where, $\mathbf{C}^{\zeta}$ is the material moduli tensor expressed in the Laplace domain as a function of the complex argument, $s$. The equation of motion in the Laplace domain is obtained by applying Eq. 6 to Eq. 1, using Eq. 7 and considering the stationary initial conditions:

$$
\sigma_{i j, j}^{\zeta}(\mathbf{x}, s)=\rho^{\zeta}(\mathbf{x}) s^{2} u_{i}^{\zeta}(\mathbf{x}, s)
$$

The kinematical relationship in the Laplace domain is:

$$
\epsilon_{i j}^{\zeta}(\mathbf{x}, s)=\frac{1}{2}\left(u_{i, j}^{\zeta}(\mathbf{x}, s)+u_{j, i}^{\zeta}(\mathbf{x}, s)\right)
$$


and the boundary conditions are:

$$
\begin{gathered}
u_{i}^{\zeta}(\mathbf{x}, s)=\bar{u}_{i}(\mathbf{x}, s) ; \quad \mathbf{x} \in \Gamma^{u} \\
\sigma_{i j}^{\zeta}(\mathbf{x}, s) n_{j}=\bar{t}_{i}(\mathbf{x}, s) ; \quad \mathbf{x} \in \Gamma^{t}
\end{gathered}
$$

where, $\overline{\mathbf{u}}(\mathbf{x}, s)$ and $\overline{\mathbf{t}}(\mathbf{x}, s)$ are the prescribed displacement and traction boundary conditions in the Laplace domain.

\section{Mathematical homogenization}

In this section, the mathematical homogenization of the governing equations defined in the Laplace domain (i.e., Eqs. 9-12) is performed. Two spatial scales, denoted by $\mathbf{x}$ and $\mathbf{y}$, are considered as shown in Fig. 1. $\mathbf{x}$ and $\mathbf{y}$ represent the coordinate vectors at the macro- and microscales, respectively. The two coordinate vectors are related to each other as $\mathbf{y}=\mathbf{x} / \zeta$, where $\zeta(0<\zeta<1)$ is the scaling factor. In the homogenization of static problems where inertial effects are small, the scaling factor is typically defined as the ratio between the characteristic length of the microstructural cell (e.g., representative volume or unit cell) and the size of the structural domain. In dynamic problems of interest, the length of the pertinent deformation and stress waves are smaller than the structural domain size, and dictate the scaling factor. The scaling factor is therefore defined as the ratio between the microstructure cell size and the length of the pertinent deformation or stress waves.

Let $f^{\zeta}(\mathbf{x}, s)$ be the Laplace transform of an arbitrary function defined in the time domain, $f^{\zeta}(\mathbf{x}, t)$. The Laplace transform of the function is expressed in terms of the two spatial scales as:

$$
f^{\zeta}(\mathbf{x}, s)=f(\mathbf{x}, \mathbf{y}(\mathbf{x}), s)
$$

The spatial derivative of $f^{\zeta}(\mathbf{x}, s)$ is obtained using the chain rule:

$$
f_{, x_{i}}^{\zeta}(\mathbf{x}, s)=f_{, x_{i}}(\mathbf{x}, \mathbf{y}, s)+\frac{1}{\zeta} f_{, y_{i}}(\mathbf{x}, \mathbf{y}, s)
$$

where, subscript comma followed by $x_{i}$ and $y_{i}$ denote the spatial derivative with respect to the macro- and microscale variables, respectively. The response fields are assumed to be spatially periodic over the characteristic volume throughout the deformation process:

$$
f(\mathbf{x}, \mathbf{y}, s)=f(\mathbf{x}, \mathbf{y}+\mathbf{k} \hat{\mathbf{y}}, s)
$$

where, $\hat{\mathbf{y}}$ denotes the period of microstructure; and $\mathbf{k}$ is a $n_{\mathrm{sd}} \times n_{\mathrm{sd}}$ diagonal matrix with integer components and $n_{\text {sd }}$ the number of spatial dimensions.

We start with the asymptotic expansion of the displacement field based on the scaling factor, $\zeta$ :

$$
u_{i}^{\zeta}(\mathbf{x}, s)=u_{i}(\mathbf{x}, \mathbf{y}, s)=u_{i}^{0}(\mathbf{x}, s)+\zeta u_{i}^{1}(\mathbf{x}, \mathbf{y}, s)+\zeta^{2} u_{i}^{2}(\mathbf{x}, \mathbf{y}, s)+\zeta^{3} u_{i}^{3}(\mathbf{x}, \mathbf{y}, s)+O\left(\zeta^{4}\right)
$$


The expansion above that is performed on the transformed displacement field is equivalent to the asymptotic expansion of the displacement field in the time domain. The physical meaning of the scaling factor, $\zeta$, is retained since the Laplace transform only affects the temporal distribution of the field. The leading order displacement term, $\mathbf{u}^{0}$, is a function of the macroscopic coordinate vector, $\mathbf{x}$, only and the higher order displacement terms depend on both the macro- and microscopic scales. Applying the displacement expansion above to Eq. 11, the strain is expressed as follows:

$$
\epsilon_{i j}(\mathbf{x}, \mathbf{y}, s)=\epsilon_{i j}^{0}(\mathbf{x}, \mathbf{y}, s)+\zeta \epsilon_{i j}^{1}(\mathbf{x}, \mathbf{y}, s)+\zeta^{2} \epsilon_{i j}^{2}(\mathbf{x}, \mathbf{y}, s)+O\left(\zeta^{3}\right)
$$

where, the strains at each order of $\zeta$ are expressed as:

$$
\begin{gathered}
\epsilon_{i j}^{\alpha}(\mathbf{x}, \mathbf{y}, s)=e_{x i j}\left(\mathbf{u}^{\alpha}(\mathbf{x}, \mathbf{y}, s)\right)+e_{y i j}\left(\mathbf{u}^{\alpha+1}(\mathbf{x}, \mathbf{y}, s)\right) ; \quad \alpha=0,1,2 \ldots \\
e_{\xi i j}\left(\mathbf{u}^{\alpha}\right)=u_{\left(i, \xi_{j}\right)}^{\alpha}(\mathbf{x}, \mathbf{y}, s)=\frac{1}{2}\left(u_{i, \xi_{j}}^{\alpha}(\mathbf{x}, \mathbf{y}, s)+u_{j, \xi_{i}}^{\alpha}(\mathbf{x}, \mathbf{y}, s)\right) ; \quad \xi=x, y
\end{gathered}
$$

Substituting Eq. 17 into Eq. 9, the stress field is obtained in the series form:

$$
\sigma_{i j}(\mathbf{x}, \mathbf{y}, s)=\sigma_{i j}^{0}(\mathbf{x}, \mathbf{y}, s)+\zeta \sigma_{i j}^{1}(\mathbf{x}, \mathbf{y}, s)+\zeta^{2} \sigma_{i j}^{2}(\mathbf{x}, \mathbf{y}, s)+O\left(\zeta^{3}\right)
$$

in which, the stress component at each order of $\zeta$ is expressed as:

$$
\sigma_{i j}^{\alpha}(\mathbf{x}, \mathbf{y}, s)=C_{i j k l}(\mathbf{y}, s) \epsilon_{k l}^{\alpha}(\mathbf{x}, \mathbf{y}, s) ; \quad \alpha=0,1,2, \ldots
$$

Due to the periodic assumption of microstructures in the problem domain, elastic moduli and density depend on the microscopic coordinate vector, $\mathbf{y}$, only (i.e. $\rho^{\zeta}(\mathbf{x})=\rho(\mathbf{y})$ and $\left.\mathbf{C}^{\zeta}(\mathbf{x}, s)=\mathbf{C}(\mathbf{y}, s)\right)$. Substituting Eqs. 16 and 20 to Eq. 10, and collecting the terms of equal orders yield the equilibrium equations at each order of $\zeta$ :

$$
\begin{aligned}
O\left(\zeta^{-1}\right): & \sigma_{i j, y_{j}}^{0}(\mathbf{x}, \mathbf{y}, s)=0 \\
O(1): & \sigma_{i j, x_{j}}^{0}(\mathbf{x}, \mathbf{y}, s)+\sigma_{i j, y_{j}}^{1}(\mathbf{x}, \mathbf{y}, s)=\rho(\mathbf{y}) u_{i}^{0}(\mathbf{x}, s) s^{2} \\
O(\zeta): & \sigma_{i j, x_{j}}^{1}(\mathbf{x}, \mathbf{y}, s)+\sigma_{i j, y_{j}}^{2}(\mathbf{x}, \mathbf{y}, s)=\rho(\mathbf{y}) u_{i}^{1}(\mathbf{x}, \mathbf{y}, s) s^{2} \\
O\left(\zeta^{2}\right): & \sigma_{i j, x_{j}}^{2}(\mathbf{x}, \mathbf{y}, s)+\sigma_{i j, y_{j}}^{3}(\mathbf{x}, \mathbf{y}, s)=\rho(\mathbf{y}) u_{i}^{2}(\mathbf{x}, \mathbf{y}, s) s^{2}
\end{aligned}
$$

The classical homogenization models rely on the first order effect of the microscale (i.e., $O(\zeta))$ on the overall response of the composite. In transient dynamics, the micro-inertia effects that results in dispersive behavior are present in the higher order equilibrium equations. To account for the dispersive behavior, we therefore include the high order terms in the formulation of the dispersive-dissipative homogenization model. 


\subsection{Homogenization procedure}

The general structure of the asymptotic analysis applied in the Laplace domain largely follows the procedure applied in the time domain, with the exception that the response fields are complex valued and functions of the complex argument, $s$. The details of the high order homogenization in the time domain is provided in Ref. [13]. In this section, the principal equations in the Laplace domain are provided.

The first order displacement term, $\mathbf{u}^{1}$, as a function of both $\mathbf{x}$ and $\mathbf{y}$, is expressed in the following form using the separation of variables:

$$
u_{i}^{1}(\mathbf{x}, \mathbf{y}, s)=U_{i}^{1}(\mathbf{x}, s)+H_{i k l}(\mathbf{y}, s) e_{x k l}\left(\mathbf{u}^{0}(\mathbf{x}, s)\right)
$$

where, $\mathbf{H}$ is the first order influence function of the microscale. $\mathbf{H}$ is a $3^{\text {rd }}$ rank tensor with the symmetry on the second and third indices (i.e. $H_{i k l}=H_{i l k}$ ) only. The microscopic balance equation is derived by substituting Eq. 23 to Eq. 22a and considering the balance within the microstructure domain, $\Theta$, as:

$$
\left\{C_{i j k l}(\mathbf{y}, s)\left(h_{k l m n}(\mathbf{y}, s)+I_{k l m n}\right)\right\}_{, y_{j}}=0 ; \quad \mathbf{y} \in \Theta
$$

in which $h_{i j m n}(\mathbf{y}, s)=H_{\left(i, y_{j}\right) m n}(\mathbf{y}, s)$ is the first order polarization function. The local periodicity of the first order displacement term, $\mathbf{u}^{1}$, implies that the first order influence function is also periodic. In addition, the normalization condition is enforced to ensure a unique solution for $\mathbf{H}$ :

$$
\left\langle H_{i k l}(\mathbf{y}, s)\right\rangle=\frac{1}{|\Theta|} \int_{\Theta} H_{i k l}(\mathbf{y}, s) d \mathbf{y}=0
$$

in which $\langle\cdot\rangle=\frac{1}{|\Theta|} \int_{\Theta} \cdot d \mathbf{y}$ denotes the averaging operator, and $|\Theta|$ is the volume of $\Theta$. By ensuring that the average of the influence function vanishes, the rigid body modes are eliminated from the solution. Eqs. 24 and 25 together with the periodic boundary conditions uniquely determine $\mathbf{H}$.

The homogenized equation of motion at $O(1)$ is obtained by applying the averaging operator on Eq. $22 \mathrm{~b}$ and exploiting the local periodicity of $\boldsymbol{\sigma}^{1}$ :

$$
\rho_{0} u_{i}^{0}(\mathbf{x}, s) s^{2}=D_{i j m n}^{0}(s) e_{x m n}\left(\mathbf{u}^{0}\right)_{, x_{j}} ; \quad \mathbf{x} \in \Omega
$$

where $\rho_{0}=\langle\rho\rangle$ is the volume-averaged density; and

$$
\begin{gathered}
D_{i j m n}^{0}(s)=\left\langle C_{i j m n}^{0}(\mathbf{y}, s)\right\rangle \\
C_{i j m n}^{0}(\mathbf{y}, s)=C_{i j k l}(\mathbf{y}, s)\left(h_{k l m n}(\mathbf{y}, s)+I_{k l m n}\right)
\end{gathered}
$$

in which, $\mathbf{D}^{0}$ is the zeroth order homogenized modulus tensor in the Laplace domain and $\mathbf{I}$ 
the fourth rank identity tensor.

We proceed with the analysis of the $O(\zeta)$ balance equations. $\mathbf{u}^{2}$ is approximated by introducing the second order influence function, $\mathbf{P}(\mathbf{y}, s)$, as:

$$
u_{i}^{2}(\mathbf{x}, \mathbf{y}, s)=U_{i}^{2}(\mathbf{x}, s)+H_{i k l}(\mathbf{y}, s) e_{x k l}\left(\mathbf{U}^{1}\right)+P_{i j k l}(\mathbf{y}, s) e_{x k l}\left(\mathbf{u}^{0}\right)_{, x_{j}}
$$

where, $\mathbf{P}$ is a fourth rank tensor and symmetric with respect to the last two indices, but not necessarily with respect to the first two indices (i.e., $P_{i j k l} \neq P_{j i k l}$ and $P_{i j k l} \neq P_{k l i j}$ ) for arbitrary microstructural configurations. Substituting Eq. 29 into Eq. 22b and considering Eq. 29, the microscopic equation of motion at $O\left(\zeta^{0}\right)$ becomes:

$$
C_{i j p m n, y_{j}}^{1}(\mathbf{y}, s)=\theta(\mathbf{y}) D_{i p m n}^{0}(s)-C_{i p m n}^{0}(\mathbf{y}, s) ; \quad \mathbf{y} \in \Theta
$$

and

$$
C_{i j p m n}^{1}(\mathbf{y}, s)=C_{i j k l}\left\{p_{k l p m n}(\mathbf{y}, s)+H_{k m n}(\mathbf{y}, s) \delta_{l p}\right\}
$$

where, $\theta(\mathbf{y})=\rho(\mathbf{y}) / \rho_{0} ; p_{k l p m n}=P_{\left(k, y_{l}\right) p m n}$; and $\boldsymbol{\delta}$ is the Kronecker delta. The local periodicity and the normalization conditions are employed in the same manner as for $\mathbf{H}$. The homogenized equation of motion at $O(\zeta)$ is derived by substituting Eqs. 23 and 29 to Eq. 22c and applying the averaging operator, in addition to considering the local periodicity of $\boldsymbol{\sigma}_{2}$ :

$$
\begin{aligned}
& \rho_{0} U_{i}^{1} s^{2}+\left\langle\rho(\mathbf{y}) H_{i k l}(\mathbf{y}, s)\right\rangle e_{x k l}\left(\mathbf{u}^{0}\right) s^{2}= \\
& D_{i j m n}^{0}(s) e_{x m n}\left(\mathbf{U}^{1}\right)_{, x_{j}}+D_{i j k m n}^{1}(s) e_{x m n}\left(\mathbf{u}^{0}\right)_{, x_{k} x_{j}} ; \quad \mathbf{x} \in \Omega
\end{aligned}
$$

in which, the first order homogenized stiffness tensor, $\mathbf{D}^{1}$, is defined as:

$$
D_{i j p m n}^{1}(s)=\left\langle C_{i j p m n}^{1}(\mathbf{y}, s)\right\rangle
$$

Next we consider the balance equation at order $O\left(\zeta^{2}\right)$. The third order displacement, $\mathbf{u}^{3}$, is approximated by introducing the third order influence function, $\mathbf{Q}(\mathbf{y}, s)$ as:

$$
\begin{aligned}
u_{i}^{3}(\mathbf{x}, \mathbf{y}, s)=U_{i}^{3}(\mathbf{x}, s)+H_{i k l}(\mathbf{y}, s) e_{x k l}\left(\mathbf{U}^{2}\right)+P_{i j k l}(\mathbf{y}, s) e_{x k l}\left(\mathbf{U}^{1}\right)_{, x_{j}} & \\
& +Q_{i j k m n}(\mathbf{y}, s) e_{x m n}\left(\mathbf{u}^{0}\right)_{, x_{k} x_{j}}
\end{aligned}
$$

Substituting Eq. 34 to Eq. 22c, the governing equation for the third order influence function, Q, after some algebra, becomes:

$$
\begin{aligned}
& C_{i j p r m n, y_{j}}^{2}(\mathbf{y}, s)=\theta(\mathbf{y}) D_{i r p m n}^{1}(s)-C_{i r p m n}^{1}(\mathbf{y}, s) \\
& +\theta(\mathbf{y})\left\{H_{i k p}(\mathbf{y}, s)-\rho_{0}^{-1}\left\langle\rho(\mathbf{y}) H_{i k p}(\mathbf{y}, s)\right\rangle\right\} D_{k r m n}^{0}(s) ; \quad \mathbf{y} \in \Theta
\end{aligned}
$$


and

$$
C_{i j p r m n}^{2}(\mathbf{y}, s)=C_{i j k l}(\mathbf{y}, s)\left\{q_{k l p r m n}(\mathbf{y}, s)+P_{k r m n}(\mathbf{y}, s) \delta_{l p}\right\}
$$

in which, $q_{k l p r m n}(\mathbf{y}, s)=Q_{\left(k, y_{l}\right) p r m n}(\mathbf{y}, s)$. The third order influence function, $\mathbf{Q}$, is a fifth rank tensor with the minor symmetry only on the last two indices (i.e. $Q_{i j k m n}=Q_{i j k n m}$ ). Since the explicit computation of $\mathbf{Q}$ is not necessary in the high order homogenization model described below, the boundary value problem for $\mathbf{Q}$ is not discussed further. Substituting Eqs. 23, 29 and 34, and applying the averaging operator to Eq. $22 \mathrm{~d}$ in addition to $\boldsymbol{\sigma}^{3}$ being locally periodic, the macroscopic homogenized equation of motion at $O\left(\zeta^{2}\right)$ is derived as:

$$
\begin{aligned}
& \rho_{0} U_{i}^{2}(\mathbf{x}, s) s^{2}+\left\langle\rho(\mathbf{y}) H_{i k l}(\mathbf{y}, s)\right\rangle e_{x k l}\left(\mathbf{U}^{1}\right) s^{2}+\left\langle\rho(\mathbf{y}) P_{i j k l}(\mathbf{y}, s)\right\rangle e_{x k l}\left(\mathbf{u}^{0}\right)_{, y_{j}} s^{2} \\
= & D_{i j m n}^{0}(s) e_{x m n}\left(\mathbf{U}^{2}\right)_{, x_{j}}+D_{i j r m n}^{1}(s) e_{x m n}\left(\mathbf{U}^{1}\right)_{, x_{r} x_{j}}+D_{i j p r m n}^{2}(s) e_{x m n}(\mathbf{u})_{,_{x_{r}} x_{p} x_{j}}^{0} ; \quad \mathbf{x} \in \Omega
\end{aligned}
$$

where, the second order homogenized stiffness tensor, $\mathbf{D}^{2}$, is expressed as:

$$
D_{i j p r m n}^{2}(s)=\left\langle C_{i j p r m n}^{2}(\mathbf{y}, s)\right\rangle
$$

The homogenized displacement field is obtained by averaging the displacement over the microstructure domain:

$$
U_{i}(\mathbf{x}, s)=\left\langle u_{i}(\mathbf{x}, \mathbf{y}, s)\right\rangle=u_{i}^{0}(\mathbf{x}, s)+\zeta U_{i}^{1}(\mathbf{x}, s)+\zeta^{2} U_{i}^{2}(\mathbf{x}, s)+O\left(\zeta^{3}\right)
$$

The summation of Eqs. 26, 32 and 37 leads to a high order homogenized equation of motion in terms of the mean displacement, $\mathbf{U}$, neglecting $O\left(\zeta^{3}\right)$ and the higher order terms:

$$
\begin{aligned}
& \rho_{0} U_{i}(\mathbf{x}, s) s^{2}+\zeta\left\langle\rho(\mathbf{y}) H_{i k l}(\mathbf{y}, s)\right\rangle e_{x k l}(\mathbf{U}) s^{2}+\zeta^{2}\left\langle\rho(\mathbf{y}) P_{i j m n}(\mathbf{y}, s)\right\rangle e_{x m n}(\mathbf{U})_{, x_{j}} s^{2}= \\
& D_{i j m n}^{0}(s) e_{x m n}(\mathbf{U})_{, x_{j}}+\zeta D_{i j k m n}^{1}(s) e_{x m n}(\mathbf{U})_{x_{k} x_{j}}+\zeta^{2} D_{i j p r m n}^{2}(s) e_{x m n}(\mathbf{U})_{x_{r} x_{p} x_{j}} ; \quad \mathbf{x} \in \Omega
\end{aligned}
$$

The terms inducing micro-inertia effects in the macroscopic equation of motion defined in Eq. 40 are scaled by the orders of $\zeta$, which leads to zero at the asymptotic limit. This appears to indicate that the contribution of the high order terms is trivial. This apparent contradiction is resolved by observing that the coefficients in these terms themselves are size dependent. It can be shown that $\mathbf{D}^{1}$ and $\langle\rho \mathbf{H}\rangle$ are proportional to $\hat{l}$, and $\mathbf{D}^{2}$ and $\langle\rho \mathbf{P}\rangle$ are proportional to $\hat{l}^{2}[3]$ :

$$
\begin{aligned}
\mathbf{D}^{1}=O(\mathbf{C} \hat{l}) ; & \langle\rho \mathbf{H}\rangle=O(\rho \hat{l}) \\
\mathbf{D}^{2}=O\left(\mathbf{C} \hat{l}^{2}\right) ; & \langle\rho \mathbf{P}\rangle=O\left(\rho \hat{l}^{2}\right)
\end{aligned}
$$

where $\hat{l}=l / \zeta$ is the characteristic length of the microstructure in the stretched coordinate system, $\mathbf{y}$, and $l$ the characteristic length of microstructure in the macroscopic coordinate 
system, x. $\mathbf{D}^{1}, \mathbf{D}^{2},\langle\rho \mathbf{H}\rangle$ and $\langle\rho \mathbf{P}\rangle$ are homogeneous functions of degree 1. Consequently:

$$
\begin{array}{cc}
\zeta \mathbf{D}^{1}=O(\mathbf{C l}) ; \quad \zeta\langle\rho \mathbf{H}\rangle=O(\rho l) \\
\zeta^{2} \mathbf{D}^{2}=O\left(\mathbf{C} l^{2}\right) ; \quad \zeta^{2}\langle\rho \mathbf{P}\rangle=O\left(\rho l^{2}\right)
\end{array}
$$

In this study, $\zeta \mathbf{D}^{1}, \zeta^{2} \mathbf{D}^{2}, \zeta\langle\rho \mathbf{H}\rangle$ and $\zeta^{2}\langle\rho \mathbf{P}\rangle$ which are directly calculated using the physical geometric size as opposed to stretched configurations. The coefficients are therefore expressed at order $O(1)$.

\subsection{A simplified high order homogenization model}

When the homogenized material is taken to exhibit orthotropy or higher symmetry, the first order homogenized moduli vanishes (i.e., $\mathbf{D}^{1}=\mathbf{0}$ ). When the moduli and density variation within the microstructure domain are spatially piecewise constant (i.e., the moduli and the density within a constituent phase is constant), it can be shown that:

$$
\left\langle\rho(\mathbf{y}) H_{i k l}(\mathbf{y}, s)\right\rangle=0
$$

Equation 43 has been demonstrated in the context of elasticity problems in the time domain by Hui and Oskay [13]. The extension to the Laplace domain is straightforward, by observing that the governing equations have identical form to the time domain equations for a fixed $s$. The complete result follows from the arbitrariness of the complex argument, $s$.

The resulting homogenized balance equation includes higher order spatial derivatives of the homogenized displacement field, $\mathbf{U}$. The standard finite element method with $C^{0}$-continuous shape functions therefore cannot be employed to evaluate this system. We seek to find an approximate simplified model which permits the use of $C^{0}$-continuous shape functions and accurately account for dispersion. We consider the following approximation of high order term:

$$
D_{\text {ijprmn }}^{2}(s) \approx \hat{A}_{i j p q}(s) D_{q r m n}^{0}(s)
$$

Since the multiplication on the right hand side of the above equation only permutes over the fourth subscript, $\mathbf{D}^{0}$ cannot be inverted to obtain $\hat{\mathbf{A}}$. Instead, we utilize the Moore-Penrose pseudo-inversion of $\mathbf{D}^{0}$ :

$$
A_{i j p q}^{*}(s)=D_{i j p r m n}^{2}(s) D_{q r m n}^{0-\mathrm{mp}}(s)
$$

where, superscript '-mp' denotes the Moore-Penrose pseudo-inverse. The Moore-Penrose inversion provides the closest approximation to $\mathbf{D}^{2}$ in the assumed form (the right hand side of Eq. 44) with respect to the Frobenius norm. The fourth order term in Eq. 40 then takes the form:

$$
\zeta^{2} D_{i j p r m n}^{2}(s) e_{x m n}(\mathbf{U})_{x_{r} x_{p} x_{j}}=\zeta^{2} A_{i j p q}^{*}(s) D_{q r m n}^{0}(s) e_{x m n}(\mathbf{U})_{, x_{r} x_{p} x_{j}}
$$


Using Eq. 26 and neglecting $O\left(\zeta^{3}\right)$ and the higher order terms:

$$
\zeta^{2} A_{i j p q}^{*}(s) D_{q r m n}^{0}(s)\left(e_{x m n}(\mathbf{U})\right)_{x_{r} x_{p} x_{j}}=\zeta^{2} \rho_{0} A_{i j m n}^{*}(s) e_{x m n}(\mathbf{U})_{, x_{j}} s^{2}
$$

Substituting Eq. 46 to Eq. 40, the macroscopic equation of motion in terms of the mean displacement, $\mathbf{U}$, becomes:

$$
\begin{aligned}
\rho_{0} U_{i}(\mathbf{x}, s) s^{2}+\zeta^{2}\left\langle\rho(\mathbf{y}) P_{i j m n}(\mathbf{y}, s)\right\rangle & \left(e_{x m n}(\mathbf{U})\right)_{, x_{j}} s^{2}= \\
& D_{i j m n}^{0}(s)\left(e_{x m n}(\mathbf{U})\right)_{, x_{j}}+\zeta^{2} \rho_{0} A_{i j m n}^{*}(s) e_{x m n}(\mathbf{U})_{, x_{j}} s^{2}
\end{aligned}
$$

The second order influence function $\mathbf{P}$ does not exhibit minor symmetry with respect to the first two indices. In order to ensure that the resulting simplified homogenized system satisfies balance of angular momentum, only the symmetric component is considered:

$$
J_{i j m n}(s)=\frac{1}{2}\left(\left\langle\rho(\mathbf{y}) P_{i j m n}(\mathbf{y}, s)\right\rangle+\left\langle\rho(\mathbf{y}) P_{j i m n}(\mathbf{y}, s)\right\rangle\right)
$$

In addition, $\mathbf{A}^{*}$ possesses the minor symmetry with respect to the first two indices only (i.e. $\left.A_{i j p q}^{*}=A_{j i p q}^{*}\right) . \mathbf{A}^{*}$ is then decomposed into its symmetric and antisymmetric components as:

$$
A_{i j k l}^{*}(s)=A_{i j k l}(s)+B_{i j k l}(s)
$$

where,

$$
\begin{aligned}
& A_{i j k l}(s)=\frac{1}{2}\left(A_{i j k l}^{*}(s)+A_{i j l k}^{*}(s)\right) \\
& B_{i j k l}(s)=\frac{1}{2}\left(A_{i j k l}^{*}(s)-A_{i j l k}^{*}(s)\right)
\end{aligned}
$$

Due to the symmetry of the strain tensor, the contribution of the antisymmetric part of $\mathbf{A}^{*}$ to the homogenized balance equation vanishes. The equation of motion for the homogenized system reduces to:

$$
\rho_{0} U_{i}(\mathbf{x}, s) s^{2}=D_{i j m n}^{0}(s)\left(e_{x m n}(\mathbf{U})\right)_{,_{j}}-L_{i j m n}(s)\left(e_{x m n}(\mathbf{U})\right)_{, x_{j}} s^{2} ; \quad \mathbf{x} \in \Omega
$$

where, the micro-inertia induced acceleration modulus tensor, $\mathbf{L}$, is defined as:

$$
L_{i j m n}(s)=\zeta^{2}\left(J_{i j m n}(s)-\rho_{0} A_{i j m n}(s)\right)
$$

The tensor, L, satisfies minor symmetry for both the first two and last two indices (i.e., $\left.L_{i j m n}=L_{j i m n}, L_{i j m n}=L_{i j n m}\right)$. From Eq. 52, the constitutive equation for the high order homogenization model at the macroscale is defined in the Laplace domain as:

$$
\Sigma_{i j}(\mathbf{x}, s)=\left(D_{i j m n}^{0}(s)-s^{2} L_{i j m n}(s)\right) e_{x m n}(\mathbf{U}) ; \quad \mathbf{x} \in \Omega
$$


in which, $\boldsymbol{\Sigma}$ denotes the homogenized stress tensor.

The errors in the proposed homogenization model include both the error introduced by the projection approximation (i.e., Eq. 44), as well as the error introduced by the homogenization assumptions. These sources of error could be separately assessed if the higher order homogenization scheme without the projection approximation is evaluated directly using higher order (i.e., $\mathrm{C}^{1}$-continuous) finite elements [22] or other numerical approaches (e.g, isogeometric analysis $[6])$. The implementation of such numerical schemes is not straightforward and out of the scope of the current manuscript. Future investigations will focus on implementation of such numerical schemes with high order continuity to better assess the impact of each error source separately.

\section{Calculation of dissipated energy}

This section provides the details of the dissipated energy calculation for the high order homogenization model. In the present work, the Prony series approximation is used in modeling the linear viscoelastic material behavior. The modulus tensor is expressed as:

$$
\begin{aligned}
g_{i j k l}(\mathbf{y}, t)=3 K_{\infty}(\mathbf{y})\left(1+\sum_{i=1}^{n} p_{i}(\mathbf{y}) e^{-t / q_{i}(\mathbf{y})}\right) & E_{i j k l}^{1}+ \\
& 2 G_{\infty}(\mathbf{y})\left(1+\sum_{i=1}^{n} p_{i}(\mathbf{y}) e^{-t / q_{i}(\mathbf{y})}\right) E_{i j k l}^{2}
\end{aligned}
$$

where:

$$
E_{i j k l}^{1}=\frac{\delta_{i j} \delta_{k l}}{3} ; \quad E_{i j k l}^{2}=\frac{\delta_{i k} \delta_{j l}+\delta_{i l} \delta_{j k}}{2}-E_{i j k l}^{1}
$$

for isotropic constituents. $p_{i}$ and $q_{i}$ are the constants of the Prony series.

$\mathbf{u}(\mathbf{x}, \mathbf{y}, s)$, is expressed in terms of the mean displacement, $\mathbf{U}(\mathbf{x}, s)$, by revisiting the asymptotic expansion of the displacement field:

$$
\begin{aligned}
u_{i}(\mathbf{x}, \mathbf{y}, s)=u_{i}^{0}(\mathbf{x}, s) & +\zeta U_{i}^{1}(\mathbf{x}, s)+\zeta^{2} U_{i}^{2}(\mathbf{x}, s)+\cdots \\
+ & \zeta H_{i k l}(\mathbf{y}, s)\left(e_{x k l}\left(\mathbf{u}^{0}\right)+\zeta e_{x k l}\left(\mathbf{U}^{1}\right)+\zeta^{2} e_{x k l}\left(\mathbf{U}^{2}\right)\right)+\cdots \\
& +\zeta^{2} P_{i j k l}(\mathbf{y}, s)\left(e_{x k l}\left(\mathbf{u}^{0}\right)_{, x_{j}}+\zeta e_{x k l}\left(\mathbf{U}^{1}\right)_{, x_{j}}+\zeta^{2} e_{x k l}\left(\mathbf{U}^{2}\right)_{, x_{j}}\right)+\cdots
\end{aligned}
$$

Rearranging the terms and using the definition of the mean displacement:

$$
u_{i}(\mathbf{x}, \mathbf{y}, s)=U_{i}(\mathbf{x}, s)+\zeta H_{i k l}(\mathbf{y}, s) e_{x k l}(\mathbf{U})+\zeta^{2} P_{i j k l}(\mathbf{y}, s) e_{x k l}(\mathbf{U})_{, x_{j}}+\cdots
$$

The strain in the Laplace domain is then calculated as:

$$
\epsilon_{i j}(\mathbf{x}, \mathbf{y}, s)=\left(I_{i j k l}+h_{i j k l}(\mathbf{y}, s)\right) e_{x k l}(\mathbf{U})+O(\zeta)
$$

in which, the presence of the influence function indicates the effect of the heterogeneity in 
the microstructure. In order to interpret and compute energy in the time domain, the strain tensor is first transformed from the Laplace domain to the time domain: $\epsilon(\mathbf{x}, \mathbf{y}, s) \rightarrow \epsilon(\mathbf{x}, \mathbf{y}, t)$.

The rate of dissipated energy density is decomposed into its deviatoric and hydrostatic components:

$$
\dot{w}(\mathbf{x}, t)=\sum_{k}^{n} \dot{w}_{s}^{k}(\mathbf{x}, t)+\sum_{k}^{n} \dot{w}_{b}^{k}(\mathbf{x}, t)
$$

where, $\dot{w}_{s}^{k}$ is the deviatoric component of the dissipated energy density rate associated with the $k^{\text {th }}$ internal viscous variable; and $w_{b}^{k}$ the hydrostatic component of the dissipated energy density associated with the $k^{\text {th }}$ internal dissipated variable. The deviatoric component is given as:

$$
\dot{w}_{s}^{k}(\mathbf{x}, t)=\frac{1}{\Theta} \int_{\Theta} 2 G_{\infty}(\mathbf{y}) \frac{p_{k}(\mathbf{y})}{q_{k}(\mathbf{y})} s_{i j}^{k} s_{i j}^{k} d \mathbf{y}
$$

in which, $\mathbf{s}^{k}$ is the deviatoric component of the dissipating strain:

$$
s_{i j}^{k}(\mathbf{x}, \mathbf{y}, t)=\int_{0}^{t} e^{-(t-\tau) / q_{k}(\mathbf{y})} \dot{\epsilon}_{i j}^{\prime}(\mathbf{x}, \mathbf{y}, \tau) d \tau
$$

where, $\boldsymbol{\epsilon}^{\prime}$ is the deviatoric component of strain. The averaging over the microstructure, $\Theta$, in Eq. 61 incorporates the dissipated energy contribution induced by the local deformation within the microstructure.

Similarly, the hydrostatic component of the dissipated energy density rate is:

$$
\dot{w}_{b}^{k}(\mathbf{x}, t)=\frac{1}{\Theta} \int_{\Theta} K_{\infty}(\mathbf{y}) \frac{p_{k}(\mathbf{y})}{q_{k}(\mathbf{y})} b_{i j}^{k} b_{i j}^{k} d \mathbf{y}
$$

in which, $\mathbf{b}^{k}$ is the hydrostatic component of the dissipated strain:

$$
b_{i j}^{k}(\mathbf{x}, \mathbf{y}, t)=\int_{0}^{t} e^{-(t-\tau) / q_{k}(\mathbf{y})} \dot{\epsilon}_{l l}(\mathbf{x}, \mathbf{y}, \tau) \delta_{i j} d \tau
$$

The total dissipated energy, $W_{d}$, is calculated by integration over the macrostructure, $\Omega$, and the time history:

$$
W_{d}(t)=\int_{0}^{t} \int_{\Omega} \dot{w}(\mathbf{x}, t) d \mathbf{x} d t
$$

\section{Numerical implementation}

The finite element formulation and implementation of the governing microscale and macroscale problems (i.e., Eqs. 24, 30 and 52) are performed to numerically evaluate the first and second order influence functions $\mathbf{H}$ and $\mathbf{P}$, as well as the homogenized displacement, $\mathbf{U}$. Periodicity (i.e., Eq.15) is enforced along the boundaries of the microstructure problems. The implementation of the periodic boundary conditions is through master-slave coupling between the corre- 
sponding boundaries. The microstructure is discretized such that nodal positions at opposing boundaries match exactly, which allows the exact imposition of the master-slave coupling and consequently the periodicity condition. Rigid body motion is eliminated by imposing zero displacement at the corner nodes. The normalization conditions on the influence functions $(\mathrm{H}$ and $\mathrm{P}$ ) are subsequently enforced by subtracting the average from each nodal value.

The equilibrium equation for the first order microscale influence function, $\mathbf{H}$, is brought to the weak form on $\mathbf{y} \in \Theta$ as:

$$
\int_{\Theta} w_{i, y_{j}}(\mathbf{y}, s) C_{i j k l}(\mathbf{y}, s) h_{k l m n}(\mathbf{y}, s) d \mathbf{y}=-\int_{\Theta} w_{i, y_{j}}(\mathbf{y}, s) C_{i j m n}(\mathbf{y}, s) d \mathbf{y}
$$

where, w $\in \mathcal{W}_{\text {per }} \subset\left[H_{\text {per }}^{1}(\Theta)\right]^{n_{\text {sd }}}$; and $H_{\text {per }}^{1}(\Theta)$ is the subspace of functions in $H^{1}(\Theta)$ that are periodic along $\Gamma_{\Theta}$, and $H^{1}(\Theta)$ is the Sobolev space of functions with square integrable derivatives. We seek the solution of the first order influence function in the finite dimensional space, $\mathbf{H} \in \mathcal{H}_{\text {per }}(\Theta) \subset\left[H_{\text {per }}^{1}(\Theta)\right]^{n_{\text {sd }} \times n_{\text {sd }} \times n_{\text {sd }}}$ such that:

$$
\begin{aligned}
& \mathcal{H}_{\mathrm{per}}(\Theta):=\left\{\mathbf{H}(\mathbf{y}, s) \mid H_{i k l}(\mathbf{y}, s)=\right. \\
&\left.\sum_{A=1}^{M} N^{[A]}(\mathbf{y}) H_{i k l}^{[A]}(s) ; H_{i k l}^{[A]}(s) \text { is } \Theta \text {-periodic; }\left\langle H_{i k l}(\mathbf{y}, s)\right\rangle=0 ; H_{i k l}^{[A]}(s)=H_{i l k}^{[A]}(s)\right\}
\end{aligned}
$$

Similarly, the weak form of the governing equation for the second order influence function problem reads:

$$
\begin{aligned}
\int_{\Theta} w_{i, y_{j}}(\mathbf{y}, s)\left(C _ { i j k l } ( \mathbf { y } , s ) \left(p_{k l p m n}(\mathbf{y}, s)+\right.\right. & \left.\left.H_{k m n}(\mathbf{y}, s) \delta_{l p}\right)\right) d \mathbf{y}= \\
& -\int_{\Theta} w_{i}(\mathbf{y}, s)\left(\theta(\mathbf{y}) D_{i p m n}^{0}(s)-C_{i p m n}^{0}(\mathbf{y}, s)\right) d \mathbf{y}
\end{aligned}
$$

for any weight function, $\mathbf{w} \in \mathcal{W}_{\text {per }}(\Theta)$. The discretization for the second order influence function belongs to the following finite dimensional space, $\mathbf{P} \in \mathcal{P}_{\text {per }}(\Theta) \subset\left[H_{\mathrm{per}}^{1}(\Theta)\right]^{n_{\mathrm{sd}} \times n_{\mathrm{sd}} \times n_{\mathrm{sd}} \times n_{\mathrm{sd}}}$ :

$$
\begin{aligned}
\mathcal{P}_{\text {per }}(\Theta):=\left\{\mathbf{P}(\mathbf{y}, s) \mid P_{i j m n}(\mathbf{y}, s)=\sum_{A=1}^{M} N^{[A]}(\mathbf{y}) P_{i j m n}^{[A]}(s) ;\right. \\
\left.P_{i j m n}^{[A]}(s) \text { is } \Theta \text {-periodic; }\left\langle P_{i j m n}(\mathbf{y}, s)\right\rangle=0 ; P_{i j m n}^{[A]}(s)=P_{i j n m}^{[A]}(s)\right\}
\end{aligned}
$$

At the macroscale, the weak form of Eq. 52 is derived as:

$$
\begin{array}{rl}
\int_{\Omega} \rho_{0} w_{i}(\mathbf{x}, s) U_{i}(\mathbf{x}, s) s^{2} & d \mathbf{x}-\int_{\Omega} w_{i, x_{j}}(\mathbf{x}, s) L_{i j m n}(s) e_{x m n}(\mathbf{U}) s^{2} d \mathbf{x} \\
+\int_{\Omega} w_{i, x_{j}}(\mathbf{x}, s) D_{i j m n}^{0}(s) e_{x m n}(\mathbf{U}) d \mathbf{x}=\int_{\Gamma^{t}} w_{i}(\mathbf{x}, s) \Sigma_{i j}(\mathbf{x}, s) n_{j} d \mathbf{x}
\end{array}
$$


for any weight function $\mathbf{w}$. The solution approximation for the mean displacement belongs to the following finite dimensional space: $\mathbf{U} \in \mathcal{U}(\Omega)$

$$
\mathcal{U}(\Omega):=\left\{\mathbf{U}(\mathbf{x}, s) \mid U_{i}(\mathbf{x}, s)=\sum_{[C]=1}^{K} N^{[C]}(\mathbf{x}) U_{i}^{[C]}(s) ; U_{i}^{[C]}(s)=\bar{u}_{i}^{[C]}(s) \text { when } \mathbf{x} \in \Gamma^{u}\right\}
$$

where $N^{[C]}$ denotes the shape function of node $C$ within the discretization of $\Omega$, and $U_{i}^{[C]}$ the nodal displacement and $K$ the total number of nodes.

Substituting the discretization of the influence and weight functions into Eqs. 66, 68 and 70 leads to the discretized forms of the macro- and microscopic problems. For a fixed complex argument, $s$, the finite element discretization and the solution procedure for the three problems has been discussed in Ref. [13] (with the exception that the current problems involve complex variables) and skipped in this manuscript. In what follows, we describe the solution strategy for the evaluation of the multiscale system.

Given the convergence region of the inverse Laplace transform integration

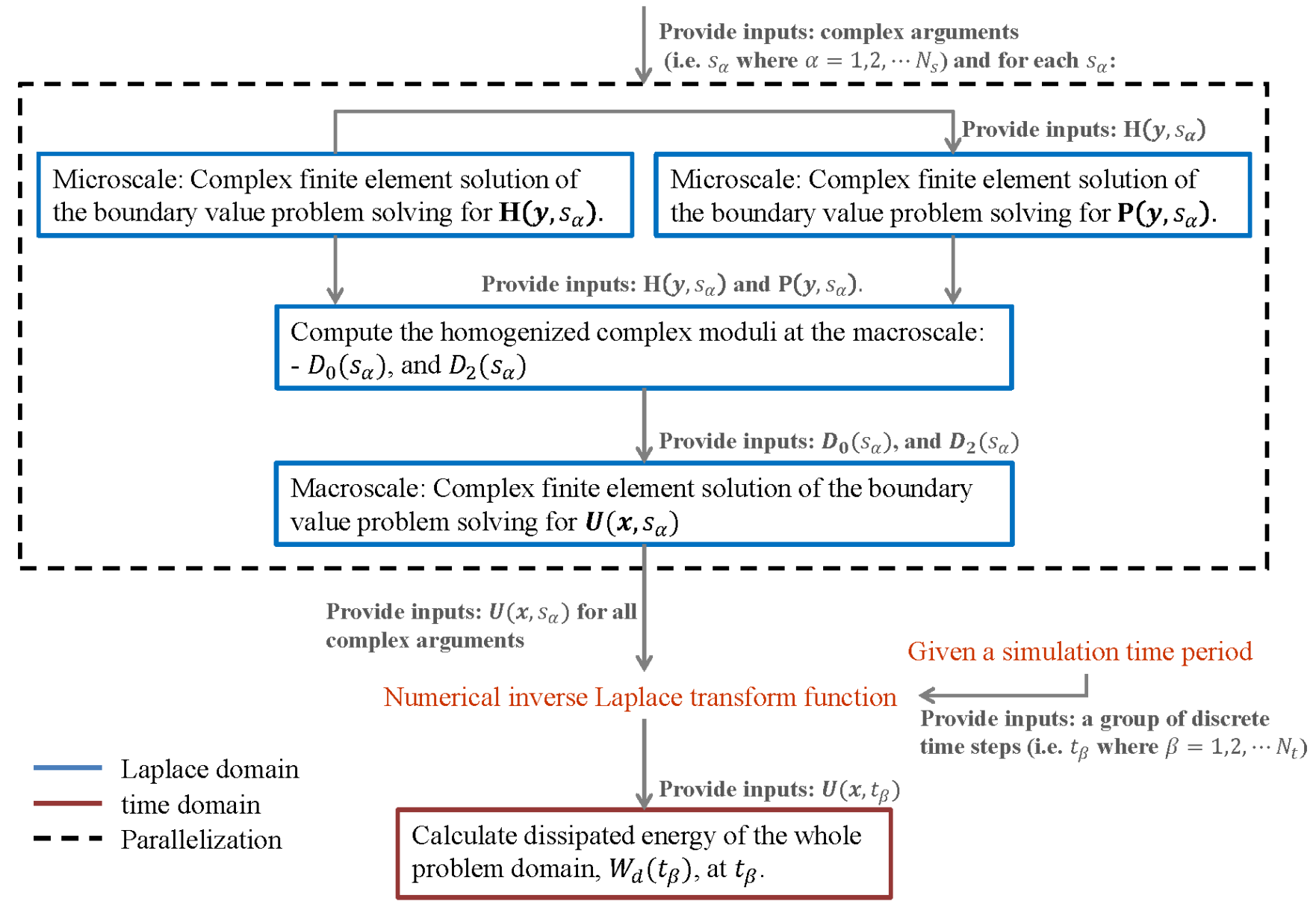

Figure 2: Computational flowchart.

The overall implementation strategy is shown in Fig. 2. The numerical implementation 
of the multiscale homogenization model includes the computation of response fields in the Laplace domain and the transformation of the Laplace domain response fields to the time domain. A numerical inverse Laplace transform [4] based on the Fast Fourier Transform and the $\epsilon$-algorithm [15] is used to transform the solutions from the Laplace domain to the time domain. The transformation is performed through several steps described below:

1. A set of discrete time steps (i.e., $t_{\beta}$ where $\beta=1,2, \cdots N_{t}$ and $N_{t}$ is the number of time steps) are generated within the given observation period. A set of complex arguments (i.e., $s_{\alpha}$ where $\alpha=1,2, \cdots N_{s}$ and $N_{s}$ is the number of complex arguments) are generated along a vertical line (i.e., of the same real part) in the complex plane and within the convergence region of the inverse Laplace transform integration [4]. The density and coverage of the generated complex arguments along the vertical line are chosen large enough for accurate inverse Laplace transform.

2. The first order influence function at $s_{\alpha}, \mathbf{H}\left(\mathbf{y}, s_{\alpha}\right)$, is obtained using the finite element solution of the boundary value problem defined by Eq. 24 in the $O(1)$ homogenization and the local periodicity and normalization conditions of $\mathbf{H}\left(\mathbf{y}, s_{\alpha}\right)$. The weak form of the governing equations is defined in Eq. 66.

3. Provided with $\mathbf{H}\left(\mathbf{y}, s_{\alpha}\right)$, the second order influence function at $s_{\alpha}, \mathbf{P}\left(\mathbf{y}, s_{\alpha}\right)$, is obtained using the finite element solution of the boundary value problem defined by Eq. 30 in the $O(\zeta)$ homogenization and the local periodicity and normalization conditions of $\mathbf{P}\left(\mathbf{y}, s_{\alpha}\right)$. The weak form of the governing equations is defined in Eq. 68.

4. The homogenized modulus tensors $\mathbf{D}^{0}\left(s_{\alpha}\right)$ and $\mathbf{D}^{2}\left(s_{\alpha}\right)$ defined in Eqs. 27 and 38 are evaluated numerically based on the solutions of $\mathbf{H}\left(\mathbf{y}, s_{\alpha}\right)$ and $\mathbf{P}\left(\mathbf{y}, s_{\alpha}\right)$.

5. The mean displacement at $s_{\alpha}, \mathbf{U}\left(\mathbf{x}, s_{\alpha}\right)$, is obtained using the finite element solution of the boundary value problem defined by Eq. 52 at the macroscale together with the corresponding boundary conditions. The weak form of the governing homogenized momentum balance equation is defined in Eq. 70 .

6. Steps 2-5 are repeated $N_{s}$ times to calculate the mean displacements in the Laplace domain for each complex argument, $s_{\alpha}$. The complex valued mean displacements are provided to the numerical inverse Laplace transform and the mean displacements for all the time steps in the real time domain are calculated.

7. The dissipated energy of the whole problem domain is computed at each time step using the mean displacements in the time domain obtained in Step 6.

The computations performed in Steps 2-5 for each complex argument value are independent of each other. This allows a straightforward and ideally scalable parallelization of the computations. The implementation procedure is therefore implemented in a parallel environment, where a large set of computations that pertain to different complex Laplace argument values are concurrently conducted. The discretization of the Laplace space (i.e., the number 
of computations realized in the Laplace space) was increased as a function of the expected response frequency content. The number is chosen large enough to ensure that further increase of resolution does not significantly alter the simulation results. In all cases, the frequency resolution is chosen to be less than $1 \mathrm{~Hz}$ to ensure accuracy in capturing response frequencies. While the Laplace space is complex, the amount of computation increases with the frequency content linearly. This is because the discretization along an imaginary (vertical) line with a fixed real component is sufficient for the discrete inverse Laplace transform (see e.g., [4]).

\section{Numerical verification}

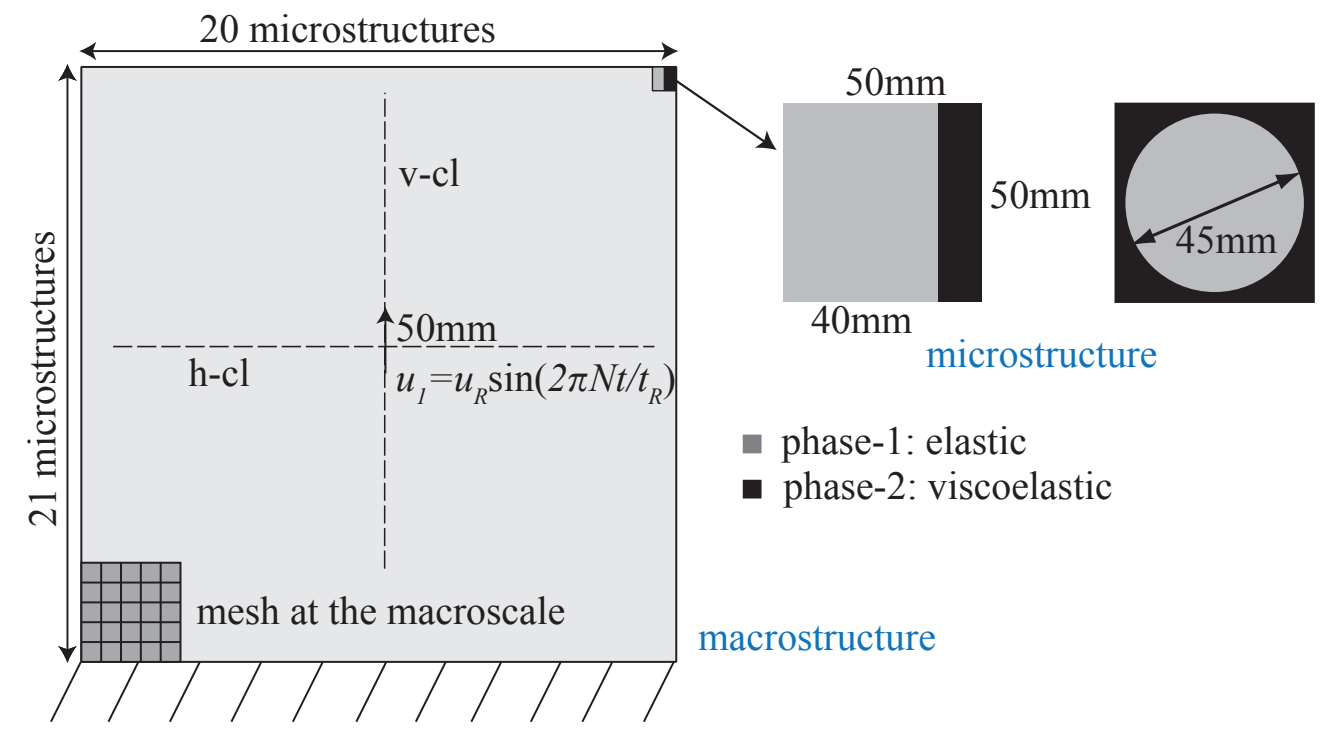

Figure 3: Layered and fibered composite structures under sinusoidal displacement disturbance.

A series of numerical simulations has been conducted to assess the performance of the proposed high order homogenization model and verified against direct numerical simulations. A two-dimensional square composite domain was considered as illustrated in Fig. 3. Two different microstructures are considered: layered and particulate. The microstructures consist of an elastic and a viscoelastic phase as shown in Fig. 3. The material parameters for the elastic and viscoelastic phases are summarized in Table 1. The volume fractions of the elastic phase in the layered and the particulate microstructures are $80 \%$ and $63.6 \%$, respectively. The composite domain was excited using a displacement controlled sinusoidal disturbance applied at the $50 \mathrm{~mm}$ segment along the vertical centerline (i.e., v-cl in Fig. 3). The maximum loading amplitude is set to $u_{R}=0.01 \mathrm{~mm}$. The domain is clamped at the bottom edge. The direct numerical simulations employed to verify the multiscale model is the direct finite element analysis (direct FEA), in which all heterogeneities are fully resolved throughout the composite domain. The direct FEA simulations use the explicit time integration with time step sizes significantly smaller than the stability limit to ensure high accuracy. 
Table 1: Material constants for elastic and viscoelastic phases.

\begin{tabular}{|c|c|c|c|}
\hline & \multicolumn{2}{|c|}{ Elastic phase } & \\
\hline$E[G P a]$ & $\nu$ & $\rho\left[\mathrm{kg} / \mathrm{m}^{3}\right]$ & \\
\hline \multirow[t]{2}{*}{2.0} & 0.3 & 7900 & \\
\hline & \multicolumn{2}{|c|}{ Viscoelastic phase } & \\
\hline$G_{\infty}[\mathrm{MPa}]$ & $K_{\infty}[\mathrm{MPa}]$ & $\rho\left[\mathrm{kg} / \mathrm{m}^{3}\right]$ & \\
\hline 22.4 & 431.4 & 1070 & \\
\hline$p_{1}$ & $p_{2}$ & $p_{3}$ & $p_{4}$ \\
\hline 0.8458 & 1.686 & 3.594 & 4.342 \\
\hline$q_{1}[\mathrm{~ms}]$ & $q_{2}[\mathrm{~ms}]$ & $q_{3}[\mathrm{~ms}]$ & $q_{4}[\mathrm{~ms}]$ \\
\hline 463.4 & 0.06407 & $1.163 \times 10^{-4}$ & $7.321 \times 10^{-7}$ \\
\hline
\end{tabular}

\subsection{Dynamic response of layered composite}

Figures 4-7 show the vertical component of the macroscopic displacement field (i.e., $U_{2}$ ) computed using the multiscale model and the direct FEA. The displacement within the problem domain is plotted at five time instances (i.e., $t=0.4,0.8,1.2,1.6$ and $2 \mathrm{~ms}$ ) for four loading frequencies: $500 \mathrm{~Hz}, 1000 \mathrm{~Hz}, 1500 \mathrm{~Hz}$ and $3000 \mathrm{~Hz}$. The domain and loading was chosen to ensure that within the simulation period $\left(t_{R}=2 \mathrm{~ms}\right)$, boundary dispersion (i.e., the interference due to reflection of the deformation waves off the domain boundaries) is relatively small and that the dispersion is largely induced by micro-heterogeneity only.

The response predictions of the multiscale model at all frequencies shown in Figs. 4-7 are in good agreement with the direct FEA. At the relatively low loading frequency of $500 \mathrm{~Hz}$ (Fig. 4), the wavelength remains well above the microstructure size and the effect of microheterogeneity induced wave dispersion is insignificant. The displacement contours show an ellipsoidal shape (e.g., Fig. 4b), since the propagations along the horizontal and vertical directions are governed by the $s$ and $p$ wave speeds, respectively $(p>s)$. When the applied loading frequency is $1000 \mathrm{~Hz}$, the interaction of the wave with the material microstructure is apparent as shown in Fig. 5. The interactions intensify at $1500 \mathrm{~Hz}$ loading frequency (Fig. 6). The wave along the horizontal centerline (h-cl in Fig. 3) is largely attenuated, which indicates the possibility of the occurrence of bandgaps. A second possible source of wave attenuation is the material dissipation due to the viscoelastic phase. The contribution of material dissipation on wave attenuation is further discussed below in terms of dissipated energies. When the loading frequency increases to $3000 \mathrm{~Hz}$ (Fig. 7), strong wave dispersion is observed and wave propagation is largely attenuated in most directions. The significance of employing the high order homogenization in capturing the band gap behavior is further verified by comparing the results to the first order homogenization method. The first order homogenization reduces to a form, where the micro-inertia induced acceleration moduli tensor, $\mathbf{L}$, is neglected in Eq. 52 . For the layered composite structure at $3000 \mathrm{~Hz}$, the first order homogenization results (Fig. 
8) indicate that the high order homogenization is clearly needed to reveal the micro-inertia effects.

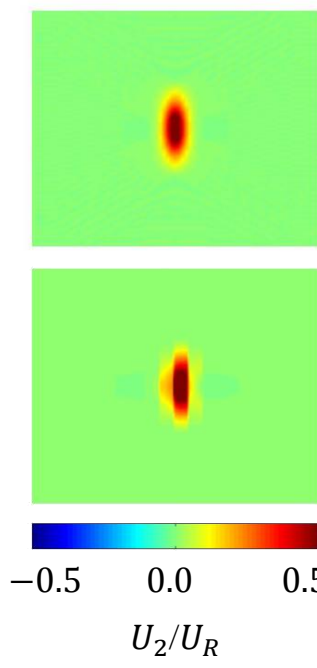

(a)
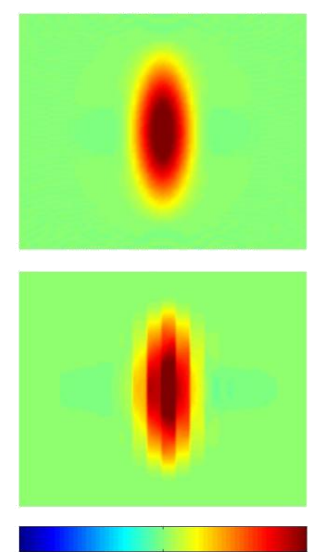
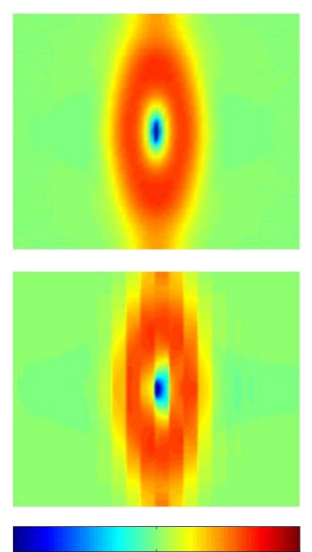
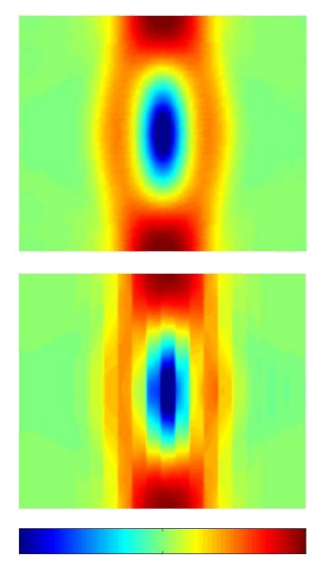

$U_{2} / U_{R}$

(c)

$$
\begin{array}{rr}
0.5-0.5 \quad 0.0 \\
\\
U_{2} / U_{R}
\end{array}
$$

(d)
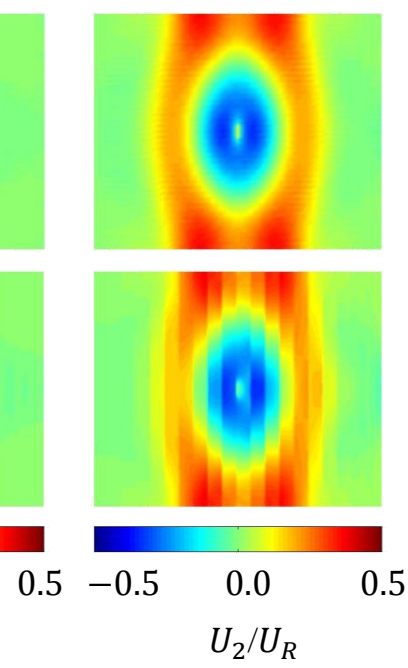

(e)

Figure 4: High order homogenization (top row) and direct FEA (bottom row) solutions when loading frequency $=500 \mathrm{~Hz}$ : (a) $\mathrm{t}=0.4 \mathrm{~ms}$; (b) $\mathrm{t}=0.8 \mathrm{~ms} ;(\mathrm{c}) \mathrm{t}=1.2 \mathrm{~ms} ;(\mathrm{d}) \mathrm{t}=1.6 \mathrm{~ms} ;(\mathrm{e}) \mathrm{t}=2 \mathrm{~ms}$.

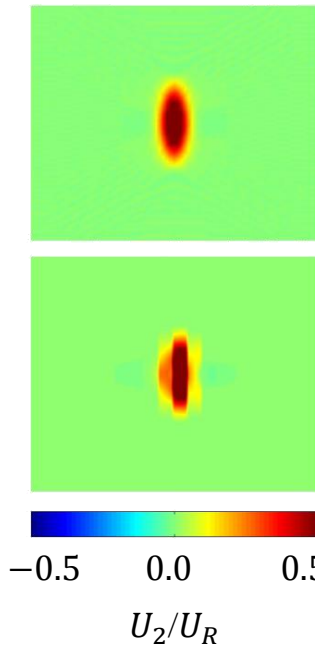

(a)
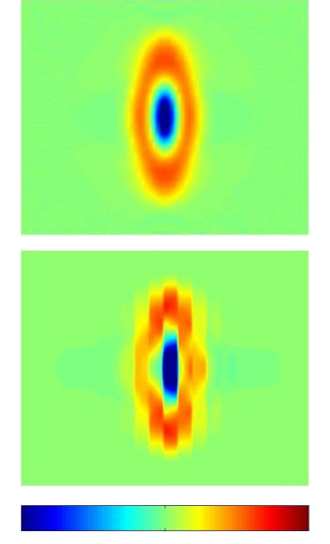
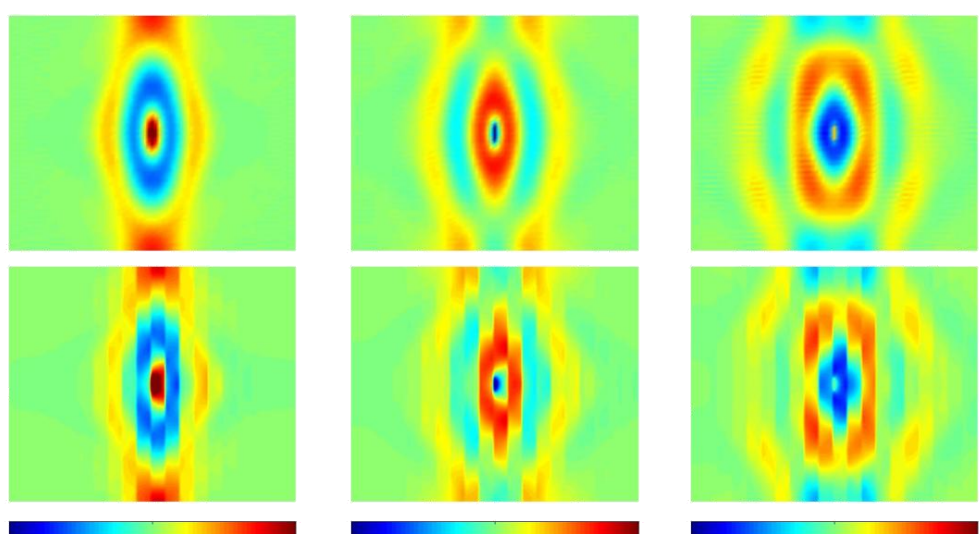

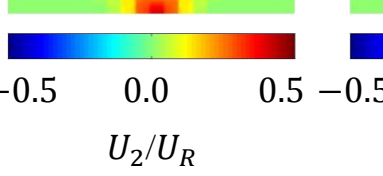

(c)

0.0
$U_{2} / U_{R}$

$(d)$

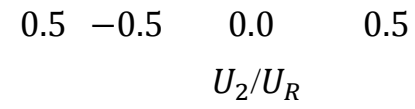

(e)

Figure 5: High order homogenization (top row) and direct FEA (bottom row) solutions when loading frequency $=1000 \mathrm{~Hz}$ : (a) $\mathrm{t}=0.4 \mathrm{~ms} ;(\mathrm{b}) \mathrm{t}=0.8 \mathrm{~ms}$; (c) $\mathrm{t}=1.2 \mathrm{~ms} ;(\mathrm{d}) \mathrm{t}=1.6 \mathrm{~ms} ;(\mathrm{e}) \mathrm{t}=2 \mathrm{~ms}$.

The occurrence of the bandgap behavior is further investigated by conducting a onedimensional shear wave propagation analysis in a layered media with identical composition to the composite domain studied herein. The one-dimensional wave propagation problem in periodic viscoelastic media has a semi analytical solution as described in Hui and Oskay [11]. 

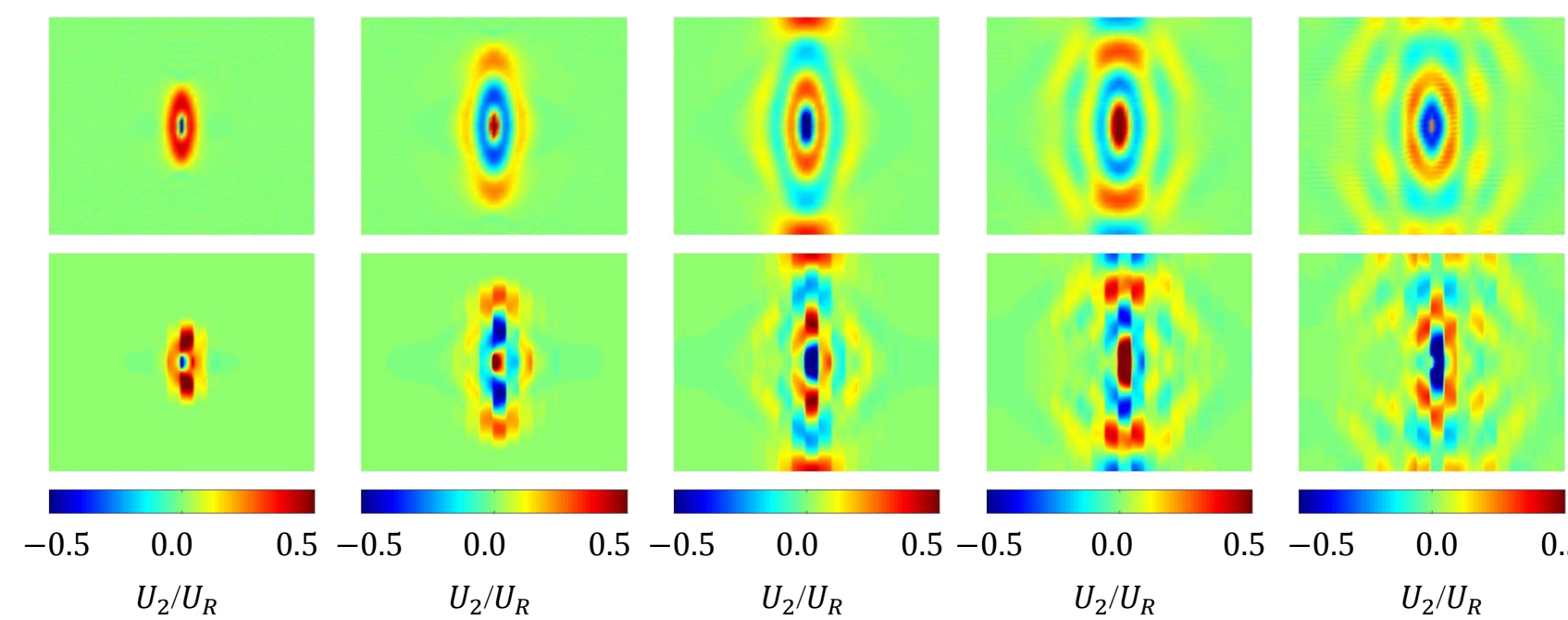

(a)

(b)
$U_{2} / U_{R}$

(c)

$$
\begin{array}{cc}
0.5-0.5 & 0.0 \\
& U_{2} / U_{R}
\end{array}
$$

(d)

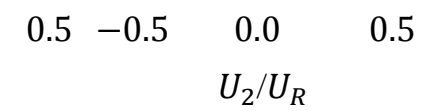

(e)

Figure 6: High order homogenization (top row) and direct FEA (bottom row) solutions when loading frequency $=1500 \mathrm{~Hz}$ : (a) $\mathrm{t}=0.4 \mathrm{~ms} ;(\mathrm{b}) \mathrm{t}=0.8 \mathrm{~ms}$; (c) $\mathrm{t}=1.2 \mathrm{~ms} ;(\mathrm{d}) \mathrm{t}=1.6 \mathrm{~ms} ;(\mathrm{e}) \mathrm{t}=2 \mathrm{~ms}$.

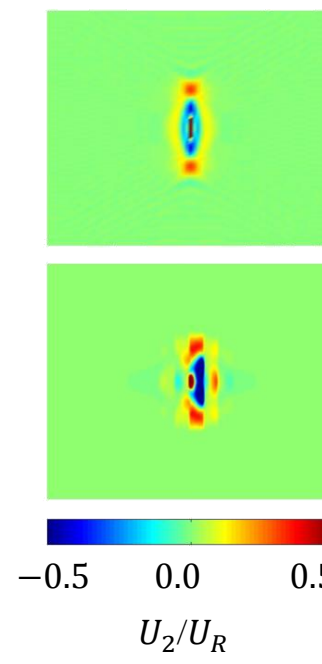

(a)
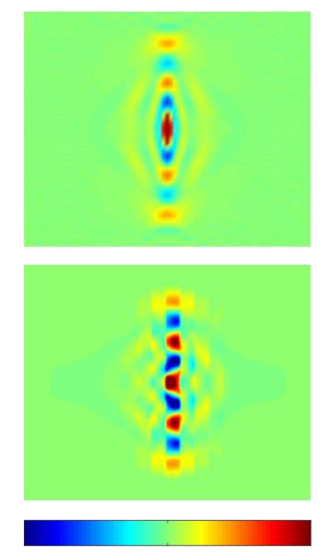

$U_{2} / U_{R}$

(b)
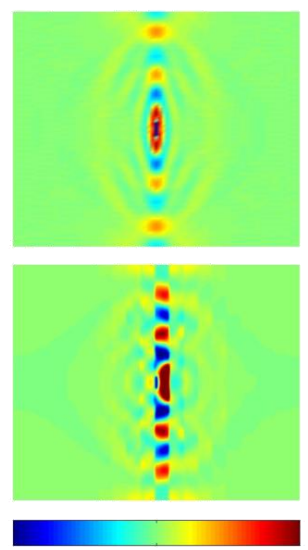
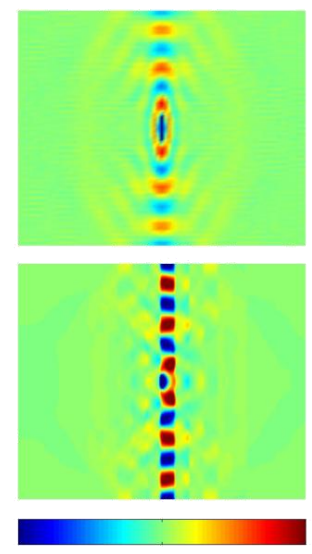

$U_{2} / U_{R}$

(c)
0.0
$U_{2} / U_{R}$

(d)
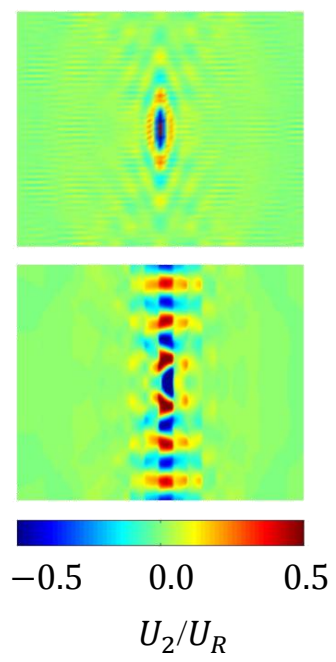

(e)

Figure 7: High order homogenization (top row) and direct FEA (bottom row) solutions when loading frequency $=3000 \mathrm{~Hz}$ : (a) $\mathrm{t}=0.4 \mathrm{~ms} ;(\mathrm{b}) \mathrm{t}=0.8 \mathrm{~ms} ;(\mathrm{c}) \mathrm{t}=1.2 \mathrm{~ms} ;(\mathrm{d}) \mathrm{t}=1.6 \mathrm{~ms} ;(\mathrm{e}) \mathrm{t}=2 \mathrm{~ms}$.

The results of the one-dimensional analysis are employed to partially verify the wave propagation in the 2 - $d$ multiscale simulations along the horizontal centerline since the propagation along the horizontal centerline is relatively undisturbed. Figure 9 shows the displacement histories of an observation point at loading frequencies of $1000 \mathrm{~Hz}, 1250 \mathrm{~Hz}$ and $1500 \mathrm{~Hz}$ as computed using the one-dimensional solution. The observation point is four microstructures (i.e., $200 \mathrm{~mm}$ ) away from the loading end. An increase in wave attenuation is observed with increasing load frequency. When the load frequency is set at $1500 \mathrm{~Hz}$ frequency, the observation 


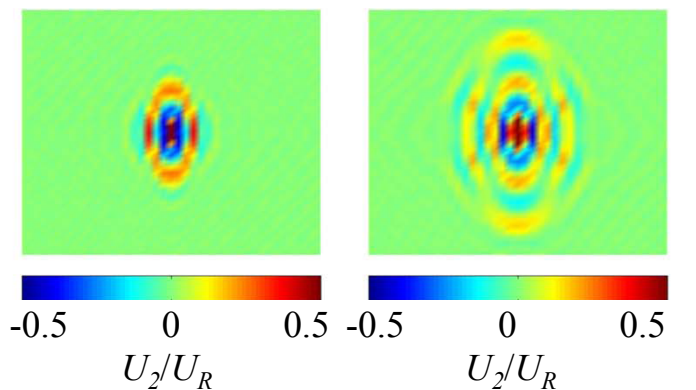

(a)

(b)

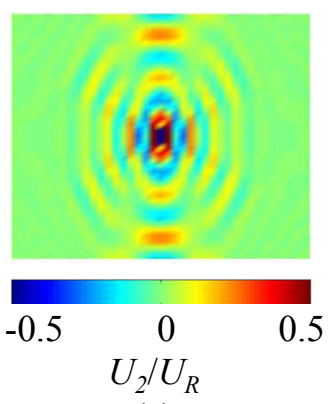

(c)

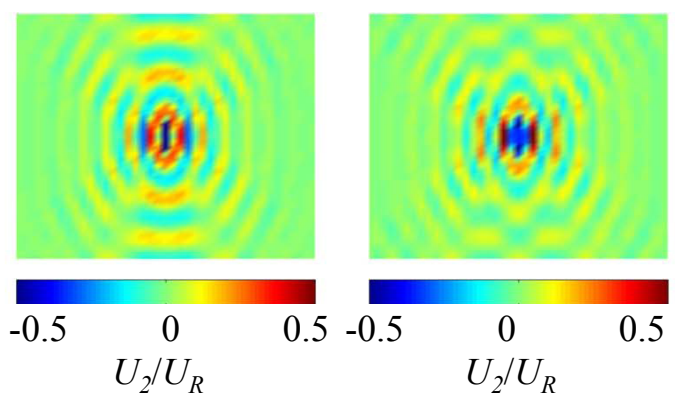

(d)

(e)

Figure 8: First order homogenization solutions when loading frequency $=3000 \mathrm{~Hz}$ : (a) t $=0.4 \mathrm{~ms}$; (b) t $=0.8 \mathrm{~ms} ;(\mathrm{c}) \mathrm{t}=1.2 \mathrm{~ms} ;(\mathrm{d}) \mathrm{t}=1.6 \mathrm{~ms} ;(\mathrm{e}) \mathrm{t}=2 \mathrm{~ms}$.

point remains undisturbed throughout the analysis period indicating either the occurrence of the bandgap or very strong material dissipation. The results of the one-dimensional simulation are in agreement with the two-dimensional simulation along the horizontal centerline.

The direct FEA solution under the loading frequency of $3000 \mathrm{~Hz}$ shows significant wave attenuation along the horizontal direction, whereas along the vertical direction localized within the central two layers, wave appears to propagate without interference. This result is consistent with the layered microstructure aligned along the vertical direction, allowing the $p$-waves to propagate freely through the uniform constituents at the mid-section. The localized vertical wave propagation is slightly damped in the multiscale model. This discrepancy is attributed to the smearing effect due to the averaging performed over the microstructure (i.e., unit cell) domain in the multiscale model, and that the plotted macroscale displacement field, $\mathbf{U}$, is the average component of the response field and the fluctuations within the microstructure domain is not shown in the multiscale solution. The discrepancy between the direct FEA and the proposed model is further demonstrated in Fig. 10, which shows the vertical component of the displacement profile along the vertical direction in the middle of the domain at $\mathrm{t}=1.6 \mathrm{~ms}$.

The effect of wave interactions with microstructure at high frequencies is reflected at the macroscale primarily through the micro-inertia induced acceleration modulus, $\mathbf{L}$. The accurate computation of the acceleration modulus depends on the discretization of the microstructure. In order to assess whether or not the microstructural mesh employed in the numerical examples is sufficiently fine, the acceleration modulus for the layered microstructure was recomputed with RVE discretizations with higher refinements. For instance, the difference between the acceleration modulus computed by the RVE mesh used in the manuscript (including 1600 elements) and a further refined mesh (including 3600 elements) is less than $0.1 \%$ (in sup norm) at arbitrary Laplace numbers. This demonstrates that the microstructural mesh employed in the numerical simulations does not significantly contribute to the model error.

The dissipated energy is calculated when the loading frequencies equal to 500, 1000, 1500 and $3000 \mathrm{~Hz}$ respectively. The dissipated energy ratio defined as the ratio of dissipated energy to total energy input is plotted in Fig. 11 where the high order homogenization solutions 


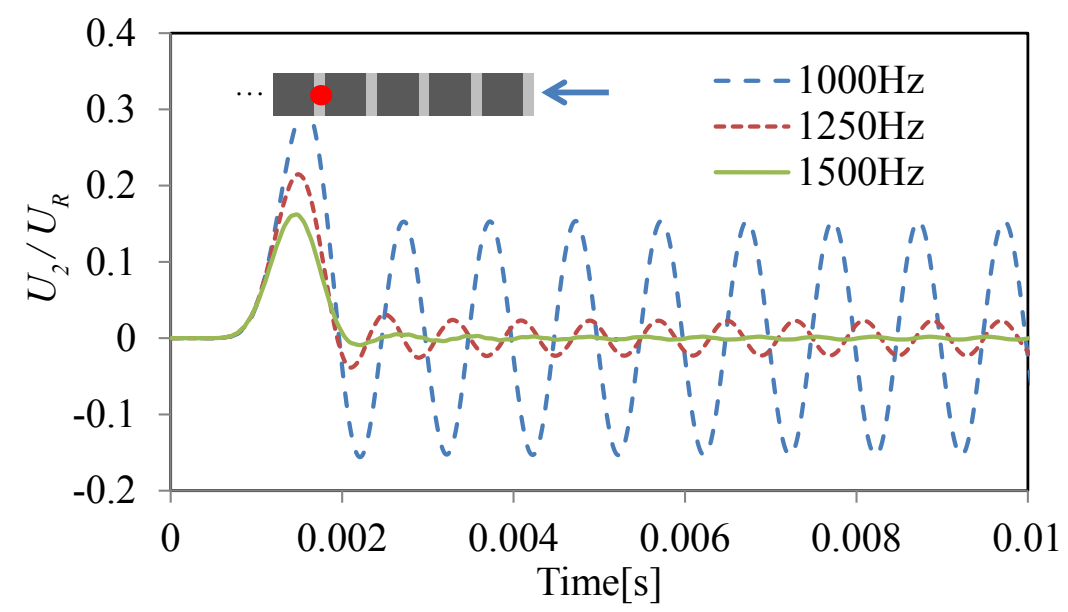

Figure 9: One-dimensional wave propagation in viscoelastic-elastic composite beam.

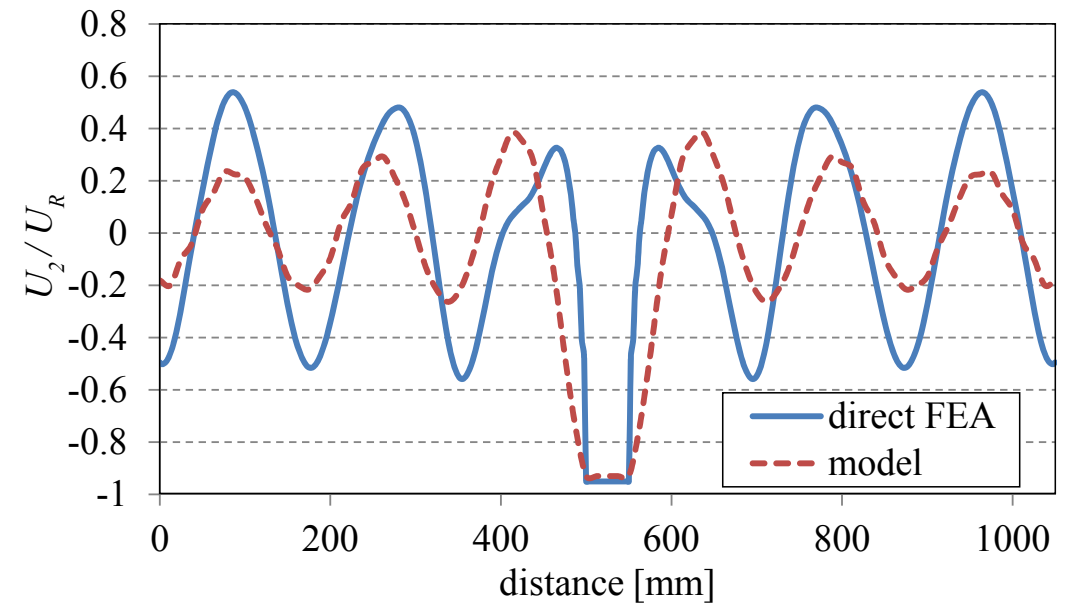

Figure 10: Vertical displacements in Fig. 7 along the vertical middle line at $t=1.6 \mathrm{~ms}$.

are compared with the direct finite element solution. The high order homogenization method predicts the dissipated energy to be in good agreement with the finite element solutions. In particular, when the loading frequency is $3000 \mathrm{~Hz}$, the high order homogenization prediction is also close to the direct finite element solution despite the discrepancy in the displacement contours as show in Fig. 7. This is because in the calculation of dissipated energy, the microscopic information (i.e., influence functions) is retrieved at each integration point at the macroscale as shown in Eq. 59. On the other hand, it is found that the dissipated energy ratio at the end of the simulation period tends to stabilize when the loading frequency increases as shown in Fig. 11. When the loading frequency is $500 \mathrm{~Hz}$, the total dissipated energy ratio is around $30 \%$; when the loading frequency increases above $1000 \mathrm{~Hz}$ until $3000 \mathrm{~Hz}$, the total dissipated energy ratio is approximately 50\%. This finding elucidates the contribution of bandgap on attenuating the wave propagation. Specifically, when the loading frequency reaches $3000 \mathrm{~Hz}$, only $50 \%$ of the total energy input is dissipated by material dissipation, and the rest of the 
input energy would make the wave propagate without the effect of microstructure induced material dispersion. In fact, as shown in Fig. 7, the wave propagation in the horizontal direction is completely blocked.
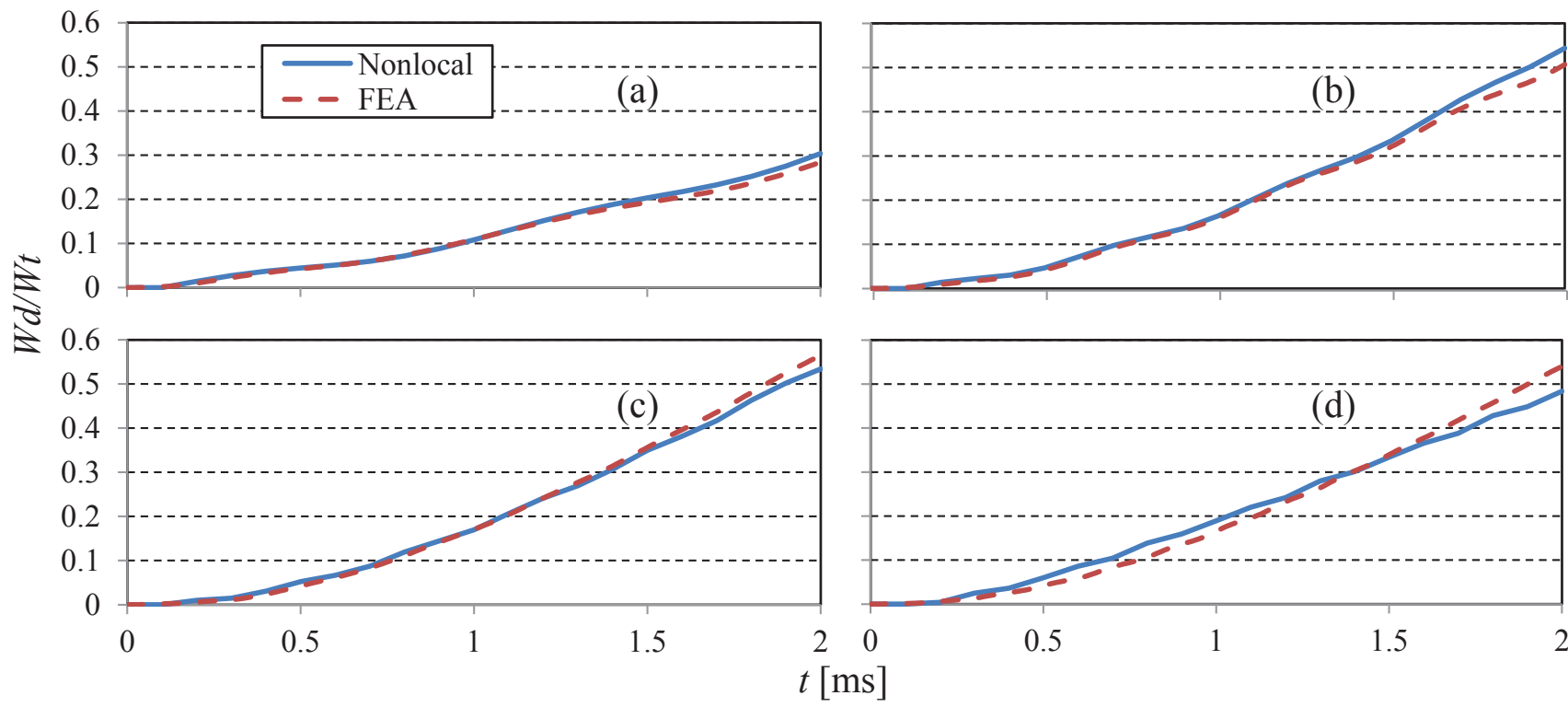

Figure 11: Dissipated energies calculated by the high order homogenization and direct FEA with loading frequency (a) $500 \mathrm{~Hz}$; (b) $1000 \mathrm{~Hz}$; (c) $1500 \mathrm{~Hz}$; (d) $3000 \mathrm{~Hz}$.

\subsection{Dynamic response of particulate composite}

The capability and the performance of the proposed multiscale model are further demonstrated by investigating the dynamic behavior of a particulate composite. The domain of the composite structure and the loading conditions remain identical to the layered configuration. The geometry of the particulate microstructure is shown in Fig. 3. The particle phase is taken to be elastic, whereas the matrix is viscoelastic with material properties shown in Table 1. Figure 12 illustrates the vertical component of the macroscopic displacement field (i.e., $U_{2}$ ) computed using the multiscale model at five time instances within the observation period (i.e., at 1.2, 2.4, 3.6, 4.8 and $6.0 \mathrm{~ms}$ ) for three different loading frequencies (i.e., $250 \mathrm{~Hz}, 500 \mathrm{~Hz}$ and $1000 \mathrm{~Hz}$ ). The wave propagation response is markedly different compared to the layered configuration due to the significantly different microstructural configurations. The loading frequency of $250 \mathrm{~Hz}$ corresponds to the wave propagation response with little material dispersion. When the loading frequency increases to $500 \mathrm{~Hz}$, strong wave dispersion occurs and the wave propagations particularly along the horizontal and vertical centerlines demonstrate significant wave attenuation. The wave propagation falls into the bandgap when the loading frequency reaches to $1000 \mathrm{~Hz}$. In contrast to the layered microstructure, near complete wave attenuation occurs at a lower frequency in the particulate composite. The sensitivity of the attenuation characteristics to the microstructure points to the capability to control the dispersion and wave 
attenuation characteristics through microstructure design. This issue will be investigated in future studies as further elaborated below.

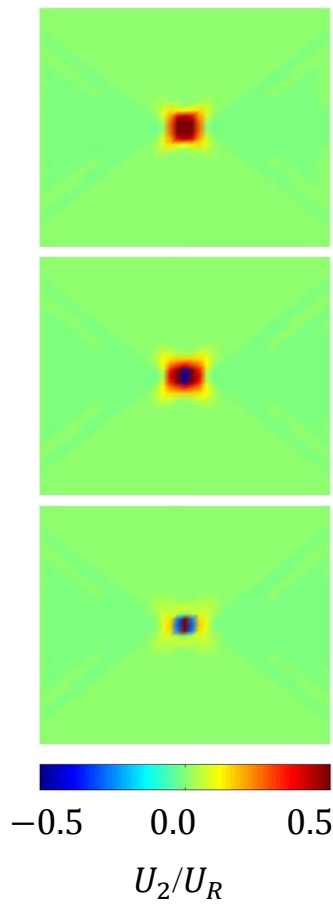

(a)
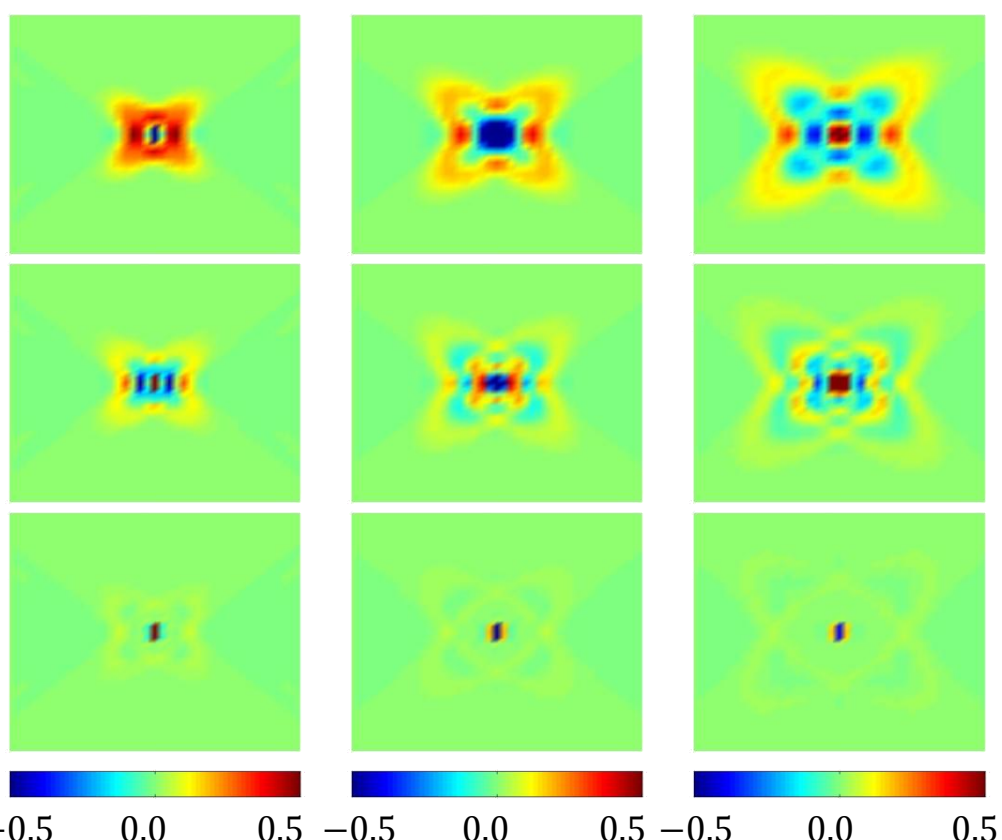

$U_{2} / U_{R}$

$(d)$
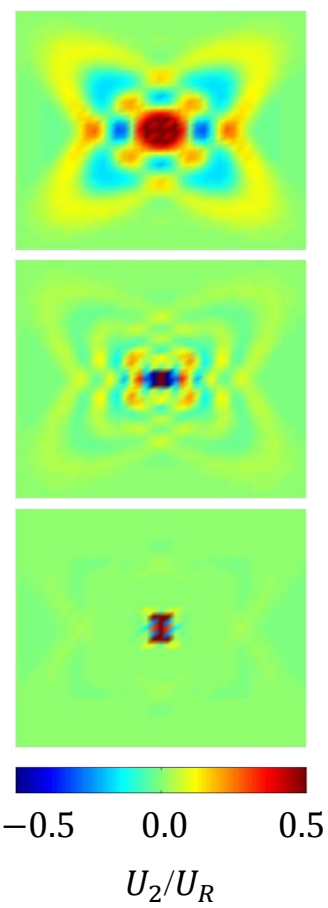

(e)

Figure 12: High order homogenization solutions when loading frequency $=250 \mathrm{~Hz}$ (top row), $500 \mathrm{~Hz}$ (middle row) and $1000 \mathrm{~Hz}$ (bottom row) at (a) t $=1.2 \mathrm{~ms}$; (b) t $=2.4 \mathrm{~ms}$; (c) t $=3.6 \mathrm{~ms}$; (d) t $=4.8 \mathrm{~ms}$; (e) $\mathrm{t}=6.0 \mathrm{~ms}$.

\subsection{Computational efficiency}

The proposed multiscale model is significantly more computationally efficient compared to the direct finite element analysis. For example of the layered composite structure, 6720 elements were used for the discretization of the macroscopic domain and 800 elements were used for the microscopic domain with the multiscale model. In comparison, 168,000 elements were used in the direct finite element solution in order to calculate the wave dispersion at high frequency loadings. For the multiscale model, both the microscopic and macroscopic problems need to be evaluated for each complex argument, s, however the tremendous reduction in the number of elements increases the computational efficiency significantly. On the other hand, 500 iterations (i.e. a set of 500 complex arguments, $s$ ) are enough to render accurate dispersive wave responses in the homogenization model while 200, 000 time steps are required to obtain accurate dispersive wave responses in the direct finite element solution. The parallelization in the homogenization model for the micro- and macroscopic problems also improves the computational efficiency in cluster environment. 


\section{Conclusions}

This manuscript presented a high order homogenization model for simulating wave propagation in viscoelastic composite structures. The proposed model is defined in the Laplace domain based on the mathematical homogenization with multiple spatial scales. The high order asymptotic terms have been introduced to capture micro-heterogeneity induced wave dispersions. The complex wave fields were solved using the finite element method with complex coefficients formulated in the Laplace domain. The performance of the proposed model was assessed by investigating wave propagation characteristics in layered and particulate composites, and verified against direct numerical simulations and analytical solutions.

The proposed model is capable of accurately predicting the wave propagations and dispersions in the layered and particulate viscoelastic composites under different loading frequencies. In addition, the multiscale model was shown to successfully predict the occurrence of and the wave propagation within the stopband. To the best of the authors' knowledge, the proposed computational model is the first computational homogenization based approach that has the ability to capture behavior within the stopbands in viscoelastic composites. The computational investigations conducted using different microstructures (i.e., layered vs. particulate) points to the sensitivity of the wave attenuation characteristics to the microstructure. This observation opens the door to the capability to control the dispersion and wave attenuation characteristics through microstructure design.

From the computational perspective, a number of challenges remain that will be addressed in the future research. First, starting from the conclusion that wave dispersion and associated wave attenuation characteristics can be controlled through the microstructure morphology, an optimization study will be performed. While many applications are possible as a function of the structure size and the frequency content, the development of microstructure design optimization framework for vibration control and impact/blast survivability in heterogeneous materials are of interest. The second challenge is the extension of the proposed model to metamaterials, which are known to exhibit stopbands through exotic properties including negative effective Poisson's ratio, negative stiffness, negative mass or a combination. The third challenge is the extension of the proposed model to account for failure processes and progressive damage accumulation. The introduction of failure processes poses significant difficulties, since the present Laplace domain homogenization procedure may not be feasible for constitutive laws that include damage and other inelastic processes.

\section{Acknowledgments}

The authors gratefully acknowledge the financial support of the National Science Foundation, CMMI Hazard Mitigation and Structural Engineering program (Grant \#:0856168). 


\section{References}

[1] I. Abu-Alshaikh, D. Turhan, and Y. Mengi. Propagation of transient out-of-plane shear waves in viscoelastic layered media. Int. J. Mech. Sci., 43:2911-2928, 2001.

[2] I. V. Andrianov, V. I. Bolshakov, V. V. Danishevs' kyy, and D. Weichert. Higher order asymptotic homogenization and wave propagation in periodic composite materials. Proc. R. Soc. London, Ser. A, 464(2093):1181-1201, 2008.

[3] C. Boutin. Microstructural effects in elastic composites. Int. J. Solids Struct., 33:10231051, 1996.

[4] L. Brancik. Programs for fast numerical inversion of laplace transforms in matlab language environment. Sbornik 7. Proc. Matlab 99, Prague, pages 27-39, 1999.

[5] E. Cosserat and F. Cosserat. Theorie des Corps Deformables. Hermann \& Fils, Paris, France., 1909.

[6] J. A. Cottrell, T. Hughes, A. Reali, and G. Sangalli. Isogeometric discretizations in structural dynamics and wave propagation. In ECOMAS Thematic Conference on Computational Methods in Structural Dynamics and Earthquake Engineering, Crete, Greece, pages $13-16,2007$.

[7] E. V. Dontsov, R. D. Tokmashev, and B. B. Guzina. A physical perspective of the length scales in gradient elasticity through the prism of wave dispersion. Int. J. Solids Struct., 50(22):3674-3684, 2013.

[8] J. Engelbrecht, A. Berezovski, F. Pastrone, and M. Braun. Waves in microstructured materials and dispersion. Philos. Mag., 85:4127-4141, 2005.

[9] C. Eringen and E. S. Suhubi. Nonlinear theory of micro-elastic solids II. Int. J. Eng. Sci, 2:189-203, 1964.

[10] S. Gonella, M. S. Greene, and W. Kam Liu. Characterization of heterogeneous solids via wave methods in computational microelasticity. J. Mech. Phys. Solids, 59:959-974, 2011.

[11] T. Hui and C. Oskay. Computational modeling of polyurea-coated composites subjected to blast loads. J. Compos. Mater., 46:2167-2178, 2012.

[12] T. Hui and C. Oskay. A nonlocal homogenization model for wave dispersion in dissipative composite materials. Int. J. Solids Struct., 50(38-48), 2013.

[13] T. Hui and C. Oskay. A high order homogenization model for transient dynamics of heterogeneous media including micro-inertia effects. Comput. Meth. Appl. Mech. Eng., 273:181-203, 2014. 
[14] M. Kafesaki, M. M. Sigalas, and E. N. Economou. Elastic wave band gaps in 3-d periodic polymer matrix composites. Solid State Commun., 96(5):285-289, 1995.

[15] J. R. Macdonald. Accelerated convergence, divergence, iteration, extrapolation, and curve fitting. J. Appl. Phys., 35:3034-3041, 1964.

[16] B. Merheb, P. A. Deymier, M. Jain, M. Aloshyna-Lesuffleur, S. Mohanty, A. Berker, and R. W. Greger. Elastic and viscoelastic effects in rubber/air acoustic band gap structures: A theoretical and experimental study. J. Appl. Phys., 104(6):064913, 2008.

[17] R. D. Mindlin. Micro-structure in linear elasticity. Arch. Ration. Mech. Anal., 16:51-78, 1964.

[18] R. P. Moiseyenko and V. Laude. Material loss influence on the complex band structure and group velocity in phononic crystals. Phys. Rev. B: Condens. Matter, 83(6):064301, 2011.

[19] T. Naciri, P. Navi, and A. Ehrlacher. Harmonic wave propagation in viscoelastic heterogeneous materials part i: Dispersion and damping relations. Mech. Mater., 18:313-333, 1994.

[20] A. H. Nayfeh. Discrete lattice simulation of transient motions in elastic and viscoelastic composites. Int. J. Solids Struct., 10:231-242, 1974.

[21] J. H. Oh, Y. J. Kim, and Y. Y. Kim. Wave attenuation and dissipation mechanisms in viscoelastic phononic crystals. J. Appl. Phys., 113(10):106101, 2013.

[22] S. A. Papanicolopulos, A. Zervos, and I. Vardoulakis. A three-dimensional c1 finite element for gradient elasticity. International journal for numerical methods in engineering, 77(10):1396-1415, 2009.

[23] K. Pham, V. G. Kouznetsova, and M .G. D. Geers. Transient computational homogenization for heterogeneous materials under dynamic excitation. J. Mech. Phys. Solids, 61 (11):2125-2146, 2013.

[24] I. E. Psarobas. Viscoelastic response of sonic band-gap materials. Phys. Rev. B: Condens. Matter, 64(1):012303, 2001.

[25] J. Sanahuja. Effective behaviour of ageing linear viscoelastic composites: Homogenization approach. Int. J. Solids Struct., 50(19):2846-2856, 2013.

[26] F. Santosa and W. W. Symes. A dispersive effective medium for wave propagation in periodic composites. SIAM J. Appl. Math., 51:984-1005, 1991. 
[27] M. M. Sigalas and E. N. Economou. Elastic waves in plates with periodically placed inclusions. J. Appl. Phys., 75(6):2845-2850, 1994.

[28] T. Suzuki and P. K. L. Yu. Tunneling in photonic band structures. J. Opt. Soc. Am. B, 12(5):804-820, 1995.

[29] T. Suzuki and P. K. L. Yu. Complex elastic wave band structures in three-dimensional periodic elastic media. J. Mech. Phys. Solids, 46(1):115-138, 1998.

[30] T. C. T. Ting. The effects of dispersion and dissipation on wave propagation in viscoelastic layered composities. Int. J. Solids Struct., 16:903-911, 1980.

[31] A. B. Tran, J. Yvonnet, Q. C. He, C. Toulemonde, and J. Sanahuja. A simple computational homogenization method for structures made of linear heterogeneous viscoelastic materials. Comput. Meth. Appl. Mech. Eng., 200(45):2956-2970, 2011.

[32] L. Tsai and V. Prakash. Structure of weak shock waves in 2-d layered material systems. Int. J. Solids Struct., 42:727-750, 2005.

[33] J. O. Vasseur, B. Djafari-Rouhani, L. Dobrzynski, M. S. Kushwaha, and P. Halevi. Complete acoustic band gaps in periodic fibre reinforced composite materials: the carbon/epoxy composite and some metallic systems. J. Phys. Condens. Matter, 6(42):8759, 1994.

[34] Z. P. Wang and C. T. Sun. Modeling micro-inertia in heterogeneous materials under dynamic loading. Wave Motion, 36:473-485, 2002.

[35] E. Yablonovitch and K. M. Leung. Photonic band structure: Non-spherical atoms in the face-centered-cubic case. Physica B, 175(1):81-86, 1991.

[36] E. Yablonovitch, T. J. Gmitter, and K. M. Leung. Photonic band structure: The facecentered-cubic case employing nonspherical atoms. Phys. Rev. Lett., 67(17):2295, 1991.

[37] Y. P. Zhao and P. J. Wei. The band gap of 1d viscoelastic phononic crystal. Comput. Mater. Sci., 46(3):603-606, 2009. 